\title{
Evolutionary state of magnetic chemically peculiar stars $\mathbf{s}^{\star \star \star}$
}

\author{
O. Kochukhov ${ }^{1}$ and S. Bagnulo ${ }^{2}$ \\ 1 Department of Astronomy and Space Physics, Uppsala University, 75120 Uppsala, Sweden \\ e-mail: oleg@astro.uu.se \\ 2 European Southern Observatory, Casilla 19001, Santiago 19, Chile
}

Received 26 November 2005 / Accepted 16 January 2006

\section{ABSTRACT}

Context. The photospheres of about 5-10\% of the upper main sequence stars exhibit remarkable chemical anomalies. Many of these chemically peculiar (CP) stars have a global magnetic field, the origin of which is still a matter of debate.

Aims. We present a comprehensive statistical investigation of the evolution of magnetic CP stars, aimed at providing constraints to the theories that deal with the origin of the magnetic field in these stars.

Methods. We have collected from the literature data for 150 magnetic CP stars with accurate Hipparcos parallaxes. We have retrieved from the ESO archive 142 FORS1 observations of circularly polarized spectra for 100 stars. From these spectra we have measured the mean longitudinal magnetic field, and discovered 48 new magnetic CP stars (five of which belonging to the rare class of rapidly oscillating Ap stars). We have determined effective temperature and luminosity, then mass and position in the H-R diagram for a final sample of 194 magnetic CP stars.

Results. We found that magnetic stars with $M>3 M_{\odot}$ are homogeneously distributed along the main sequence. Instead, there are statistical indications that lower mass stars (especially those with $M \leq 2 M_{\odot}$ ) tend to concentrate in the centre of the main sequence band. We show that this inhomogeneous age distribution cannot be attributed to the effects of random errors and small number statistics. Our data suggest also that the surface magnetic flux of CP stars increases with stellar age and mass, and correlates with the rotation period. For stars with $M>3 M_{\odot}$, rotation periods decrease with age in a way consistent with the conservation of the angular momentum, while for less massive magnetic CP stars an angular momentum loss cannot be ruled out.

Conclusions. The mechanism that originates and sustains the magnetic field in the upper main sequence stars may be different in CP stars of different mass.

Key words. stars: chemically peculiar - stars: evolution - stars: fundamental parameters - stars: magnetic fields

\section{Introduction}

Observations suggest that magnetic fields are ubiquitous in late-type stars, and that a correlation exists between magnetic activity and stellar rotation. The magnetic field of late-type stars is typically localised in spots, and evolves on relatively short time scales. Although not yet fully understood, a dynamo mechanism is commonly invoked to explain the presence of a magnetic field in these kinds of stars.

Early-type stars show a completely different magnetic phenomenology. Magnetic fields appear organised on a large scale at the stellar surface, and do not change within a time scale shorter than several decades. Instead, a periodic field variability is observed, which is commonly interpreted in terms of the so-called Oblique Rotator Model: the magnetic field geometry is not symmetric about the rotation axis, and the observer

^ Tables 1 and 2 are only available in electronic form at http://www.edpsciences.org

$\star \star$ Based on observations made with ESO Telescopes at the Paranal Observatory under programs ID 71.D-0308, 72.D-0377, and 73.D0464, retrieved through the ESO archive. sees a magnetic configuration that changes as the star rotates. The field strength (typically a few hundreds up to a few tens of thousands of Gauss) does not seem correlated to the star's rotational velocity. Only a minority (about 5\%) of early-type stars is magnetic. Practically all known magnetic stars of the upper main sequence are classified between late F- and early B-type, and belong to the category of the so-called chemically peculiar (CP) stars, i.e., stars that exhibit distinctive peculiarities in the element abundance of their atmospheres. Most CP stars (hence most magnetic stars) show also abnormally slow rotation.

The origin of the magnetic fields in CP stars is a matter of debate (Moss 2004). The dynamo hypothesis can hardly explain the observed high field strengths and the lack of a correlation with rotation. A more promising approach is offered by the fossil field theory (Cowling 1945; Moss 1989; Braithwaite \& Spruit 2004), according to which the observed fields in the upper main sequence magnetic stars are the remnants of fields present during earlier stages of stellar evolution. The fact that no correlation is observed with stellar rotation, and the fact that only a small percentage of the upper main sequence stars 
are magnetic, are naturally explained in terms of variations in the amount of magnetic flux trapped during star formation. However, it not yet clear (observationally nor theoretically) if and how these fields evolve during the main sequence phase.

To provide constraints to the theory of the origin of the magnetic field, it is important to study the evolutionary state of magnetic CP stars. This has been done by several authors, and with conflicting conclusions. Based on a small sample of about 30 magnetic stars, Hubrig et al. (2000) suggested that magnetic fields appear at the surface of CP stars with $M \lesssim 3 M_{\odot}$ only after they have spent considerable fraction of their life on the main sequence. Most low mass stars studied by Hubrig et al. (2000) were drawn from the group of very slowly rotating magnetic stars with detectable Zeeman splitting in their spectra (Mathys et al. 1997). This selection procedure results in a statistic sample small in size, that may also be intrinsically biased toward older stars (should the star's angular momentum be lost or/and the surface magnetic field increase during the main sequence evolutionary phase). Both Gomez et al. (1998) and Pöhnl et al. (2005) investigated much larger samples of CP stars, but instead of selecting objects for which magnetic field was detected, they utilised chemical peculiarity as a proxy of magnetism. They have found that CP stars with chemical anomalies similar to those observed in magnetic CP stars occupy all the regions of the main sequence.

In an attempt to clarify this puzzling situation, we have carried out a new study of the evolution of magnetic stars of the upper main sequence that is based on a very large sample of observed magnetic CP stars, collected through a thoroughfull investigation of the literature and of the ESO archive. Our study includes all known objects for which precise parallaxes were measured by the Hipparcos mission, and for which the presence of magnetic field at the surface could be asserted via direct field detections. We have also investigated whether there are observational evidences for angular momentum losses occurring during the star's evolution in the main sequence.

This paper is organised as follows. Section 2 describes the observations collected from the literature, and presents new magnetic measurements obtained from the analysis of data collected with FORS1 at the ESO VLT. In Sect. 3 we determine the temperature and luminosity of the selected stellar sample, and in Sect. 4 we derive the position of the selected stars in the $\mathrm{H}-\mathrm{R}$ diagram. Results and statistical analysis are presented in Sect. 5 and discussed in Sect. 6.

\section{The stellar sample}

\subsection{Known magnetic stars}

The main part of our sample of magnetic CP stars has been drawn from various literature sources. The catalogues by Romanyuk (2000) and Bychkov et al. (2003) have provided the core of the sample of the early-type stars with definite magnetic field detections. Since the present study focuses on the classical magnetic $\mathrm{Ap} / \mathrm{Bp}$ stars, we have selected objects with the $\mathrm{SrCrEu}, \mathrm{Si}$, and/or He chemical peculiarities for which reliable detection of magnetic field has been obtained with one of the two direct methods: spectropolarimetric or photopolarimetric measurement of the disk-averaged line of sight (longitudinal) magnetic field (Mathys 1991; Borra \& Landstreet 1980) and the mean field modulus diagnostic using resolved Zeeman split spectral line profiles (Mathys et al. 1997).

The list of stars compiled from the two catalogues was supplemented by a number of recently discovered magnetic stars. In particular, the studies by Aurière et al. (2004), El'kin et al. (2003), Johnson (2004), Hubrig et al. (2003, 2005), Ryabchikova et al. (2004, 2005), Shorlin et al. (2002), and Stütz et al. (2003) have provided objects satisfying our selection criteria. We refer the reader to the original papers for the description of the respective observations and their analysis. Putting aside details of these studies of individual magnetic CP stars, it is worth noting that all investigations mentioned above relied on the classical direct methods of the magnetic field detection (spectropolarimetry and/or measurement of the resolved Zeeman split lines) and that only objects with reliable ( $>3 \sigma$ in the case of longitudinal field measurements) magnetic field detections were included in our sample.

According to the current understanding of the incidence of magnetism in early-type stars, the non-magnetic chemically peculiar stars, including objects with the HgMn and Am peculiarity types, do not host global magnetic fields similar to those found in the classical magnetic CP stars. Although claims of the detection of magnetic field in HgMn or Am stars have occasionally been made (e.g., Mathys \& Lanz 1990; Hubrig \& Castelli 2001), these studies typically employed an indirect diagnostic of magnetic field based on the analysis of magnetic broadening and intensification of spectral lines. Furthermore, none of the alleged detections of the complex fields in nonmagnetic CP stars was confirmed by a subsequent, independent observation or supported by direct spectropolarimetric magnetic field diagnostic technique (Shorlin et al. 2002; Wade et al. 2003). Given this absence of the solid evidence for the presence of significant surface fields in HgMn and Am stars, all such objects were eliminated from our list of magnetic stars. We emphasise that in nearly all cases when non-zero longitudinal field determinations are reported for $\mathrm{HgMn}$ or Am stars in the catalogues compiled by Romanyuk (2000) and Bychkov et al. (2003), the original measurements date back to the early lowprecision spectropolarimetric observations by Babcock (1958).

The magnetic stellar sample compiled in the present study was cross-matched with the Hipparcos parallax data (Perryman et al. 1997). Parallaxes with an accuracy better than $20 \%$ (which is the same threshold as employed by Hubrig et al. 2000) could be retrieved for 150 magnetic CP stars. Magnetic field in the majority of these stars was detected using polarimetric observations. Consequently, unlike the 33 magnetic stars studied by Hubrig et al. (2000), our sample does not suffer from the possible bias of over-representing the slowly rotating and strongly magnetic old stars for which resolved Zeeman split lines are observable.

\subsection{Magnetic stars observed with FORS1 at VLT}

FORS1 (FOcal Reducer/low dispersion Spectrograph) of the ESO VLT is a multi-mode instrument equipped with 
polarisation analysing optics including super-achromatic halfwave and quarter-wave phase retarder plates, and a Wollaston prism. In the last few years, FORS1 has been extensively used in polarimetric mode to measure magnetic fields in various kinds of stars, for instance in white dwarfs (e.g., Aznar Cuadrado et al. 2004), in hot subdwarfs (O'Toole et al. 2005), in the central stars of planetary nebulae (Jordan et al. 2005). In particular, several observing programs have been dedicated to the magnetic field surveys of Ap stars, and many data are now available in the ESO archive. For this work we have retrieved 142 observations of circular polarised spectra for a total of 100 Ap stars. All targets have been observed using grism $600 \mathrm{~B}$ and a $0.4^{\prime \prime}$ slit-width, which gives a spectral resolution of about 2000, covering the spectral range 3550-5850. A typical observing block consists of four frames obtained with the $\lambda / 4$ retarder waveplate oriented at $+45^{\circ}$ and four frames obtained with the retarder waveplate at $-45^{\circ}$. Data have been reduced using standard IRAF routines in order to get wavelength calibrated spectra from the ordinary and extra-ordinary beams taken with the $\lambda / 4$ retarder waveplate oriented at $+45^{\circ}$ and $-45^{\circ}$. From these spectra we have obtained Stokes $I$ and $V$ profiles using Eq. (4.1) of the FORS1+2 User Manual (VLTMAN-ESO-13100-1543), but with the sign changed. We have then considered the Stokes $I$ and $V$ profiles of the Balmer lines from $\mathrm{H} \beta$ down to the Balmer jump, and then we have obtained the longitudinal field $\left\langle B_{z}\right\rangle$ using a least-square technique based on the formula

$\frac{V}{I}=-g_{\mathrm{eff}} C_{z} \lambda^{2} \frac{1}{I} \frac{\mathrm{d} I}{\mathrm{~d} \lambda}\left\langle B_{z}\right\rangle$,

where $g_{\mathrm{eff}}$ is the effective Lande factor ( $=1$ for the hydrogen Balmer lines, see Casini \& Landi Degl'Innocenti 1994), $\lambda$ is the wavelength expressed in $\AA,\left\langle B_{z}\right\rangle$ is the longitudinal field expressed in Gauss, and

$C_{z}=\frac{e}{4 \pi m_{\mathrm{e}} c^{2}} \quad\left(\simeq 4.67 \times 10^{-13} \AA^{-1} \mathrm{G}^{-1}\right)$,

where $e$ is the electron charge, $m_{\mathrm{e}}$ the electron mass, $c$ the speed of light. Equation (1) is valid under the weak-field approximation, which, for the hydrogen Balmer lines formed in a typical atmosphere of an A-type star, holds for field strength up to $\sim 20 \mathrm{kG}$. More details on the data reduction technique, and the way $\left\langle B_{z}\right\rangle$ is calculated, are given in Bagnulo et al. (2002) and Bagnulo et al. (2005).

The new $\left\langle B_{z}\right\rangle$ measurements are reported in Table 1 (available in electronic form only). Magnetic field was detected at $>3 \sigma$ level in 53 stars. Only five of them were previously known to be magnetic, whereas the other four lack accurate parallax data in the Hipparcos catalogue. Thus, analysis of the archival FORS1 spectra has contributed with 44 magnetic stars, increasing the total sample investigated in the present paper to 194 objects.

We note that definite detection of the longitudinal field in five members (HD 19918, HD 42659, HD 60435, HD 84041, HD 86181) of the rare class of rapidly oscillating Ap (roAp) stars is reported here for the first time, thereby significantly increasing the number of roAp stars with known magnetic field properties.

\section{Determination of effective temperatures and luminosities}

\subsection{Photometric effective temperature}

Stellar effective temperatures were determined using calibration of the Geneva photometric system (Golay 1972). Observed photometric parameters were extracted from the catalogue of Rufener (1989) and supplemented by the data available through the online photometric database at Geneva Observatory ${ }^{1}$. We determined $T_{\text {eff }}$ of CP stars following the procedure suggested by Hauck \& North (1993) and revised by Hauck \& Künzli (1996). For hot stars calibration in the theoretical grids published by Künzli et al. (1997) was used in combination with the linear $T_{\text {eff }}$ correction to account for anomalous flux distribution of magnetic CP stars (Hauck \& Künzli 1996). For cool Ap stars we employed calibration of the $(B 2-G)_{0}$ color index proposed by Hauck \& North (1993).

For a few CP stars lacking photometric measurements in the Geneva system we determined $T_{\text {eff }}$ using Strömgren uvby $\beta$ photometric data (Hauck \& Mermilliod 1998) and calibration by Moon \& Dworetsky (1985).

Effective temperature of the extreme cool magnetic peculiar star HD 101065 (Przybylski's star) cannot be determined using any usual calibrations available either for normal or CP stars. Instead, a $T_{\text {eff }}=6450 \mathrm{~K}$ was adopted for this object based on the results of recent detailed spectroscopic studies (Cowley et al. 2000; Kochukhov et al. 2002). The spectroscopic $T_{\text {eff }}=7750 \mathrm{~K}$ (Kochukhov et al. 2002) was also used for HD 216018, which lacks a complete set of Strömgren or Geneva photometry.

For a subsample of stars with both Strömgren and Geneva photometry available, uncertainty of effective temperature can be estimated from the discrepancy of $T_{\text {eff }}$ values given by independent calibrations in the two different photometric systems. Based on this assessment we adopted $\sigma\left(T_{\text {eff }}\right)=200 \mathrm{~K}$ for stars with $T_{\text {eff }} \leq 8500 \mathrm{~K}, \sigma\left(T_{\text {eff }}\right)=300 \mathrm{~K}$ for $8500<T_{\text {eff }} \leq$ $10500 \mathrm{~K}, \sigma\left(T_{\text {eff }}\right)=400 \mathrm{~K}$ for $10500<T_{\text {eff }} \leq 16000 \mathrm{~K}$, and $\sigma\left(T_{\text {eff }}\right)=500 \mathrm{~K}$ for stars hotter than $T_{\text {eff }}=16000 \mathrm{~K}$. These error estimates are very similar to the $T_{\text {eff }}$ uncertainty assumed by Hubrig et al. (2000, see their Tables 1 and 2), although no explicit discussion of the adopted $T_{\text {eff }}$ error bars can be found in the latter paper.

\subsection{Correction for the interstellar extinction and reddening}

The interstellar extinction and reddening have to be taken into account for stars located farther away than $60 \mathrm{pc}$. We considered four different procedures to obtain color excess $E(B-V)$ for individual stars in our sample: from the intrinsic $[U-$ $B$ ] color and reddening-free Geneva $X$ and $Y$ parameters of hotter stars (Cramer 1982), from the interstellar extinction maps of Lucke (1978) and Schlegel et al. (1998), and using the model of Hakkila et al. (1997). The $E(B-V)$ parameters obtained from these sources were averaged after rejection of occasional outliers. Based on the scatter of the color excess values

\footnotetext{
${ }^{1}$ http://obswww. unige.ch/gcpd/ph13.html
} 



Fig. 1. Distribution of the Hipparcos parallaxes (top) and their relative errors (bottom) for the sample of magnetic CP stars.

derived using different methods, we found a typical uncertainty of $E(B-V)$ to be $0.005 \mathrm{mag}$ for $E(B-V) \leq 0.05$ and $0.010 \mathrm{mag}$ for $E(B-V)>0.05$. For several strongly reddened objects a higher $E(B-V)$ error bar had to be adopted, reflecting large standard deviation of highly discrepant reddening estimates.

Photometric parameters in the Geneva and Strömgren system were dereddened with the help of relations based on the interstellar extinction laws given by Fitzpatrick (1999). Interstellar extinction in the $V$-band was calculated using $R \equiv$ $A_{V} / E(B-V)=3.1$.

\subsection{Hipparcos luminosity}

Absolute magnitudes and luminosities of the program stars were determined on the basis of data from the Hipparcos catalogue (Perryman et al. 1997) and the $V$-magnitude information extracted from the SIMBAD database. Distribution of the Hipparcos parallaxes and their relative errors is illustrated in Fig. 1. Most of the studied stars are located within $250 \mathrm{pc}$ from the Sun, but only a few are closer than $60 \mathrm{pc}$. The average parallax uncertainty for the magnetic stars in our sample is $11 \%$. Roughly half of the stars have parallax determined with an accuracy better than that.
From the comparison of magnitudes given in different literature sources, we estimated uncertainty of $m_{V}$ related to the measurement errors and intrinsic stellar variability to be approximately $0.02 \mathrm{mag}$.

For a number of magnetic stars known to be members of spectroscopic or unresolved visual binaries we applied positive duplicity correction to $m_{V}$. Generally, this correction can be estimated from the luminosity ratios available from literature for the majority of well-studied SB2 and visual binaries. On the other hand, CP stars belonging to SB1 systems usually lack precise luminosity ratio estimates. For these objects we adopted $\Delta m_{V}=0.16$ which corresponds to the magnitude difference of 2.0 between components.

The absolute magnitude in the $V$-band was determined using the standard relation:

$M_{V}=m_{V}+5+5 \log \pi-A_{V}$,

where trigonometric parallax $\pi$ is measured in arcseconds and interstellar extinction was determined as outlined above. The error estimate for the absolute magnitude took into account uncertainties in $m_{V}, \pi$, and $A_{V}$ :

$\sigma\left(M_{V}\right)=\sqrt{\sigma^{2}\left(m_{V}\right)+\left(\frac{5 \sigma(\pi)}{\pi \ln 10}\right)^{2}+\sigma^{2}\left(A_{V}\right)}$.

Calculating the stellar luminosity,

$\log \frac{L}{L_{\odot}}=-\frac{M_{V}+B C-M_{\mathrm{bol}}(\odot)}{2.5}$,

we adopted the solar bolometric magnitude $M_{\mathrm{bol}}(\odot)=+4.75$ (Bessell 2000) and used the standard bolometric correction $B C$ taken from Flower (1996). Since in the latter paper $B C$ is tabulated as a function of $T_{\text {eff }}$, uncertainty of effective temperature contributes to the total error of luminosity that can be estimated according to the following expression:

$\sigma\left(\log \frac{L}{L_{\odot}}\right)=0.4 \sqrt{\sigma^{2}\left(M_{V}\right)+\left(\frac{\mathrm{d} B C}{\mathrm{~d} T_{\text {eff }}}\right)^{2} \sigma^{2}\left(T_{\text {eff }}\right)}$.

Taking into account all contributions to the $M_{V}$ and $L / L_{\odot}$ error budgets, we find a typical uncertainty of $20-25 \%$ for both parameters.

Anomalous flux distribution of peculiar stars is characterized by the enhanced ultraviolet absorption which induces backwarming in the visible (Leckrone 1973). This makes CP stars sub-luminous for their visual colours, and bolometric correction has to be modified accordingly (North 1981; Lanz 1984). In the present paper we use $B C$ tabulated as a function of $T_{\text {eff }}$ for normal stars (Flower 1996). However, we account for peculiar nature of CP stars in derivation of their $T_{\text {eff }}$ (Sect. 3.1). With this procedure the average change in $B C$ due to anomalous stellar flux distribution is taken into account implicitly. Remaining modifications of $B C$ depend on individual properties of magnetic stars and are difficult to estimate without detailed model atmosphere analysis. Nevertheless, this $B C$ uncertainty is likely to be much smaller compared to the average error of $M_{\mathrm{bol}}(0.24 \mathrm{mag})$ for the stars in our sample.

No Lutz-Kelker (LK, Lutz \& Kelker 1973) correction was applied to the absolute magnitudes of magnetic stars in our 
sample. The original LK correction, which is always negative and hence systematically increases luminosity estimated from parallax, has been a source of confusion and extensive debate in literature. In a recent publication Smith (2003) summarised this discussion and concluded that the LK correction is meaningful only when applied to the stellar samples, but should not be used in studies of individual stars. As pointed out by Stępień (2004), large (from $-0.1 \mathrm{mag}$ up to $-0.5 \mathrm{mag}$ ) negative LK corrections adopted by Hubrig et al. (2000) displaced the stars in their sample significantly upwards from the zero age main sequence (ZAMS), possibly resulting in apparent lack of young low mass magnetic stars. A similar problem may have affected results of Pöhnl et al. (2005) who, contrary to the recommendations of Smith (2003) and Stępień (2004), have also attempted to use the LK procedure to correct individual stellar magnitudes.

\section{Mass and age determination}

Theoretical evolutionary tracks of the upper main sequence stars are available for different metal abundance of the stellar envelope (see Schaller et al. 1992; and Schaerer et al. 1993). Large deviations of the chemical composition of CP stars from the normal solar abundance table are believed to be limited to the surface layers. These superficial chemical anomalies are produced by the process of selective radiative diffusion in the presence of magnetic field, stellar wind, and possibly weak turbulent mixing. These complex hydrodynamical effects are poorly understood and hence it is not possible to estimate the average interior metal content of individual CP stars based on the observed surface abundance pattern. In this situation a fixed, usually solar, metallicity has to be assumed to make comparison of the observed and predicted stellar parameters possible (e.g., Hubrig et al. 2000; Pöhnl et al. 2005). Here we have adopted metallicity $Z=0.018$ and obtained theoretical stellar evolutionary tracks by interpolating within the grids of Schaerer et al. (1993) and Schaller et al. (1992), who published calculations for $Z=0.008$ and $Z=0.020$, respectively. The plausible effect of the dispersion in $Z$ can be estimated from the scatter in the surface abundances determined for normal B stars and nearby young $\mathrm{F}$ and $\mathrm{G}$ stars. Using the summary of the Fe and light element abundances given by Sofia \& Meyer (2001), we obtain star-to-star $Z$ variation of $\approx 0.002$. The resulting effect on the age and mass determination of CP stars is smaller than other error sources.

Figure 2 illustrates distribution of magnetic CP stars in the theoretical H-R diagram. Each star is shown with a point, whereas respective error bars give uncertainty of $T_{\text {eff }}$ and luminosity. The ZAMS and the envelope of the lowest $T_{\text {eff }}$ achieved during the core hydrogen burning phase are also shown. Given a set of theoretical isochrones, the problem of the stellar mass determination reduces to interpolation within the evolutionary tracks tabulated for different masses. To avoid degeneracy, all stars were assumed to lie within the main sequence zone where $T_{\text {eff }}$ decreases monotonously. For a few objects located below the ZAMS or above the lowest $T_{\text {eff }}$ line, parameters were calculated for the closest main sequence point.

Subsequent determination of the stellar age is complicated by the uneven evolution of stars in the $\log T_{\text {eff }}-\log L / L_{\odot}$ plane. Young stars located close to the ZAMS change their temperature and luminosity very slowly. The pace of evolution increases rapidly as the star ages and shifts towards the terminal age main sequence. Therefore, the same uncertainty of $T_{\text {eff }}$ and $L$ translates into dramatically different age errors, depending on whether the star is young or evolved. Although this effect is well-known, to our knowledge, no attempt has ever been made to take it properly into account in the statistical studies of the evolutionary state of CP stars. In the present paper we developed a non-linear error propagation procedure to obtain realistic errors of the absolute and relative ages. For each star in our sample we determined mass and age for the point corresponding to the adopted stellar $\log T_{\text {eff }}$ and $\log L / L_{\odot}$ and then repeated this procedure for 8 positions along the error ellipse defined by the individual uncertainty of temperature and luminosity. Resulting minimum and maximum ages yield realistic asymmetric range of evolutionary stages compatible with a given pair of $T_{\text {eff }}$ and $L$ and their respective $1 \sigma$ error limits. Application of this non-linear error propagation procedure to the magnetic CP star HD 56350 is illustrated in Fig. 3.

The summary of the age confidence limits is given in Fig. 4. Here and elsewhere in the paper we quantify the relative stellar age, $\tau$, by the fraction of the stellar life spent on the main sequence. The ZAMS line corresponds to $\tau=0$, whereas the star at the end of the core hydrogen burning phase has $\tau=1$. As it follows from Fig. 4, the relative age of individual young CP stars cannot be determined with an accuracy better than $\approx 20 \%$. Only for the oldest stars in our sample the H-R diagram fitting yields ages precise at the 5-7\% level.

The final set of the stellar fundamental parameters and corresponding $1 \sigma(68 \%)$ confidence intervals is reported in Table 2 (available in electronic form only). For each star we list its identification in the HD and Hipparcos catalogues, distance, absolute magnitude, effective temperature, luminosity, mass, absolute and relative ages. Using the data in Table 2, is also straightforward to estimate the stellar radius

$\log \left(R / R_{\odot}\right)=0.5 \log \left(L / L_{\odot}\right)-2 \log \left(T_{\text {eff }} / T_{\text {eff } \odot}\right)$

and the surface gravitational acceleration

$\log \left(g / g_{\odot}\right)=\log \left(M / M_{\odot}\right)+4 \log \left(T_{\text {eff }} / T_{\text {eff } \odot}\right)-\log \left(L / L_{\odot}\right)$.

\section{Results}

\subsection{Distribution of stars in the $H$-R diagram}

We have grouped all magnetic stars in our sample into three different mass bins: stars with $M \leq 2 M_{\odot}$, stars with $2 M_{\odot}<$ $M \leq 3 M_{\odot}$, and stars with $M>3 M_{\odot}$. Figure 5 shows the distribution of the relative ages for the stars belonging to these three groups. An alternative overview of the age distribution of the magnetic stars of various masses is given in Fig. 6. We found that $26 \%$ of stars with mass $>3 M_{\odot}$ have spent less than $30 \%$ of their life in the main sequence. This percentage is $18 \%$ for stars with mass between $2 M_{\odot}$ and $3 M_{\odot}$, and only $16 \%$ for stars with $M \leq 2 M_{\odot}$. It appears thus that higher mass stars $\left(M>3 M_{\odot}\right)$ are homogeneously distributed in fractional age. In the group of stars of intermediate mass, younger stars seem 


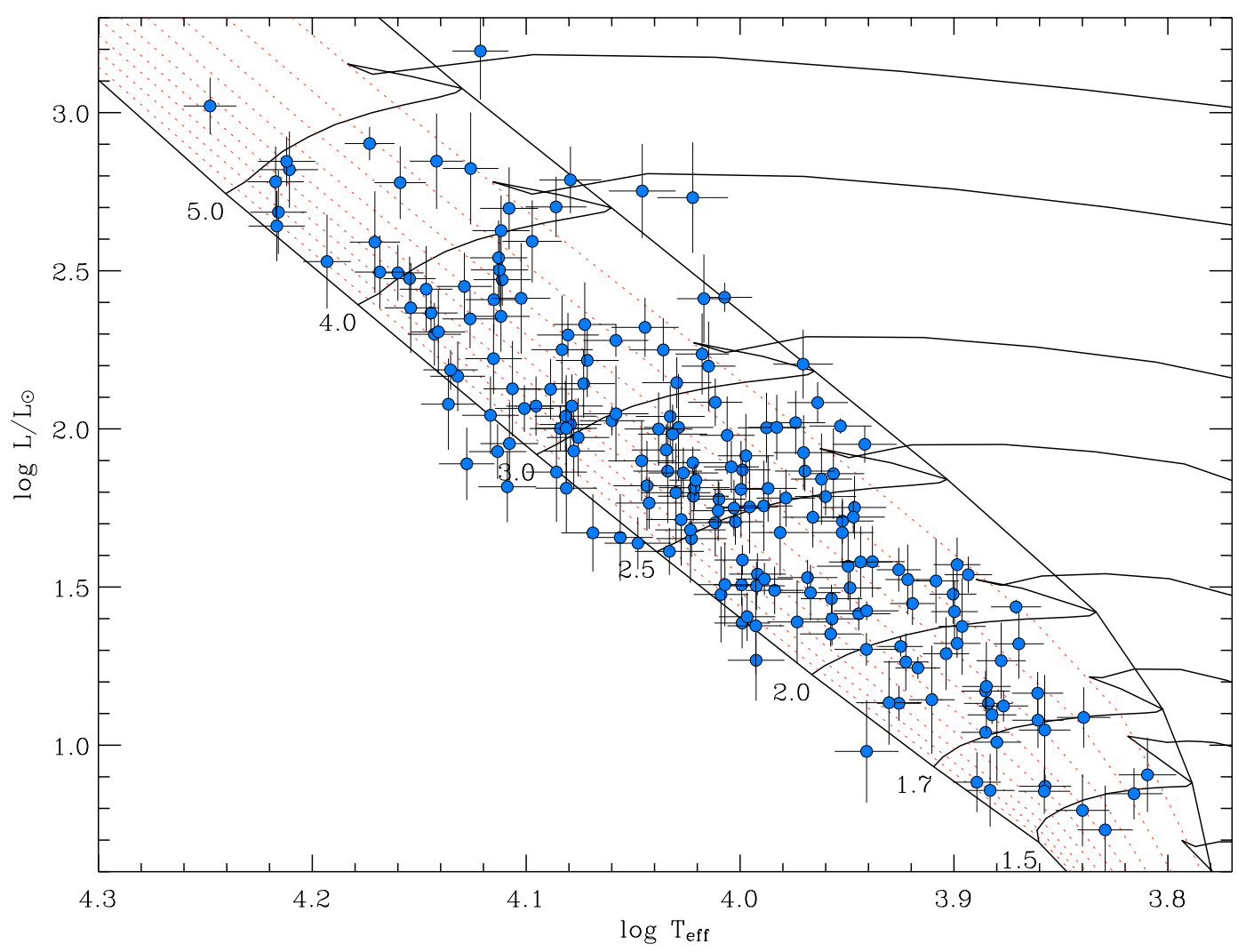

Fig. 2. Position of magnetic chemically peculiar stars on the Hertzsprung-Russell diagram. The solid lines show theoretical evolutionary tracks, the ZAMS and the envelope of lowest $T_{\text {eff }}$ achieved during the main sequence evolutionary stage (Schaller et al. 1992; Schaerer et al. 1993). Dotted curves correspond to the lines of equal fractional age $\tau$ measured in units of the main sequence stellar lifetime. These curves are plotted with a step of 0.1 between the ZAMS $(\tau=0)$ and the end of the core hydrogen burning phase $(\tau=1)$.

less numerous than older stars. Among stars with $M \leq 2 M_{\odot}$ the shortage of young objects is even more pronounced.

The statistical validity of these results must be carefully investigated, since a priori one could argue that a small sample of objects randomly selected from a homogeneous group may well be characterized by special features that in fact are not representative of the entire sample. In other words, one could suspect that the apparent shortage of young magnetic stars with $M \leq 3 M_{\odot}$ is just the result of a statistical fluctuation. It should also be noted that young stars are those for which age determination is less accurate. In order to clarify this situation, we have performed numerical simulations to calculate what is the probability that, repeating the same study, one obtains a number of stars with $\tau<0.3$ equal or smaller than what we have found. This estimates the false alarm probability, i.e. the chance that the observed uneven distribution of stellar ages can be attributed to a statistical fluctuation.

First of all, we have given an analytical representation to the error bars of the fractional age, by using a linear fit to the values given in Table 2. Namely, we have estimated the lower and upper error bars as

$\Delta \tau_{-}=0.33-0.29 \tau$ and $\Delta \tau_{+}=0.27-0.26 \tau$,

respectively. Then we have considered a sample of $N$ objects. To each of them, we have associated a random number $p_{i}$ from 0.0 to 1.0, representing the fractional age. Each $p_{i}$ number has been transformed to a $p_{i}^{\prime}=p_{i}+\delta\left(p_{i}\right)$ value, where the "errors" $\delta\left(p_{i}\right)$ were deduced from a Gaussian distribution with standard deviation $\Delta \tau_{-}$(for $\delta(p)<0$ ) or $\Delta \tau_{+}$(for $\delta(p)>0)$, using again a random number generator. All $p_{i}^{\prime}<0$ have been then set to 0 , and all $p_{i}^{\prime}>1$ have been changed to 1 . We have then counted the number $J_{k}$ of $p_{i}^{\prime}$ values included within the interval $[0.0,0.3]$. We have repeated the same exercise $M$ times, and finally counted the number $L$ of times in which $J_{k}$ was equal or smaller than a certain number $Q$. The ratio $P_{1}(N, Q)=L / M$ gives the probability that, in a sample of $\mathrm{N}$ stars homogeneously distributed in fractional age, we find no more than $Q$ stars in the interval [0.0,0.3].

In our observational sample of $N=32$ magnetic stars with $M \leq 2 M_{\odot}$, we have found only 5 stars with $\tau \leq 0.3$. Therefore we have performed the statistical test described above using $N=32$ and $Q=5$, and calculated $P_{1}(32,5)=6.5 \%$. We have also found that in the sample of 93 magnetic stars with $2 M_{\odot}<M \leq 3 M_{\odot}, 17$ have $\tau \leq 0.3$. For this case, the statistical test gives $P_{1}(93,17)=1.3 \%$. Finally, we have found that 18 over 69 magnetic stars with $M>3 M_{\odot}$ have $\tau \leq 0.3$, and we have calculated $P_{1}(69,18)=36 \%$.

It is also of interest to test a complementary hypothesis that magnetic stars belong to a homogeneous population of objects with a relative age of at least $\tau=0.3$, and all young stars in our sample appear entirely due to observational errors. In order to investigate this possibility, we have repeated the 


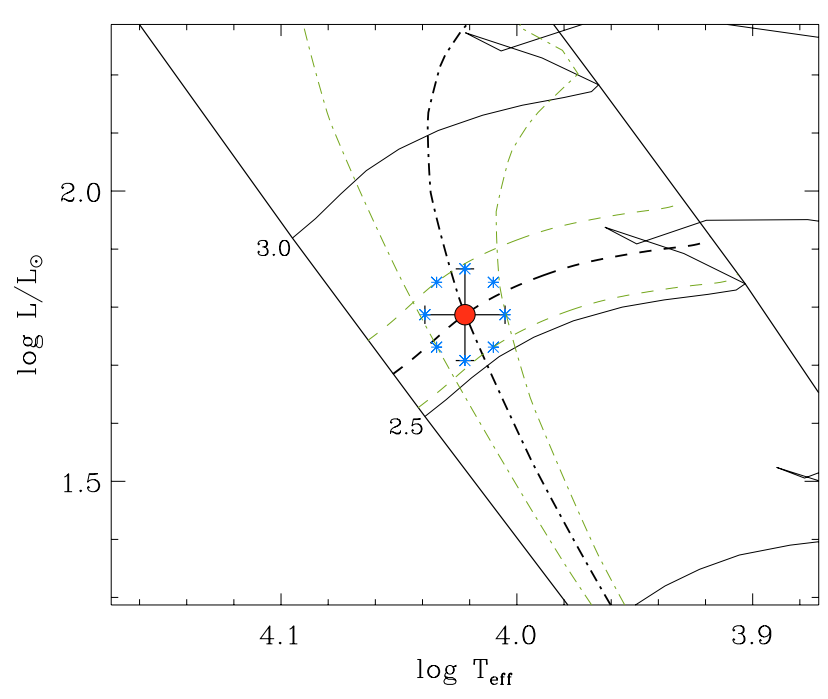

Fig. 3. Illustration of the error analysis applied to derive asymmetric confidence intervals for the stellar mass and age. The solid lines show theoretical evolutionary tracks (Schaller et al. 1992; Schaerer et al. 1993). The point and error bars indicate our estimate of the temperature and luminosity of the magnetic CP star HD 56350. Thick dashed and dash-dotted lines correspond to interpolated evolutionary tracks and isochrones, respectively. Interpolation procedure is repeated for 8 locations along the error ellipse in the $\log T_{\text {eff }}-\log L / L_{\odot}$ plane, as shown by asterisks. Thin dashed and dash-dotted curves show tracks and isochrones for minimum and maximum mass and age. This analysis yields a stellar mass $M / M_{\odot}=2.610_{-0.088}^{+0.090}$ and age $\log t=8.40_{-0.21}^{+0.11} \mathrm{yr}$.

simulations choosing the fractional age randomly in the interval $[0.3,1.0]$ and counting trials in which the number stars with $\tau<0.3$ has reached the observed value. The resulting probability, $P_{2}(N, Q)$, turns out to be considerable for stars with $M \leq 2 M_{\odot}\left(P_{2}=23 \%\right)$ but is negligible $\left(P_{2} \lesssim 1 \%\right)$ for more massive stars.

Another conspicuous feature of the stellar age distributions presented in Figs. 5 and 6 is the relatively small number of $M \leq 3 M_{\odot}$ stars at the end of their main sequence life. Stellar evolution is fast in this region of the H-R diagram. Consequently, the relative age is determined with good precision (see Fig. 4). Applying the same statistical approach as outlined above (for stars in the $[0.0,1.0]$ age interval) we could, however, verify that the lack of stars with $\tau \geq 0.8$ is not particularly significant by itself: the corresponding false alarm probability, $P_{3}(N, Q)$ is $7 \%$ and $40 \%$ for the two groups of low mass stars $\left(M \leq 2 M_{\odot}\right.$ and $2 M_{\odot}<M \leq 3 M_{\odot}$, respectively), and $P_{3}=81 \%$ for stars with $M>3 M_{\odot}$.

We have further extended the statistical analysis to test the possibility that the overall shape of the observed distribution of stellar ages can be attributed to random errors and effects of small number statistics. This was achieved by computing the composite probability that, given the number of objects observed in each mass range, the fraction of stars in the relative age interval $[0.0,0.4]$ (the sum of the first two bins in the histograms of Fig. 5) and, simultaneously, of those in the $[0.8,1.0]$ interval (the last bin in Fig. 5) does not exceed the observed values. We have found that the observed strong
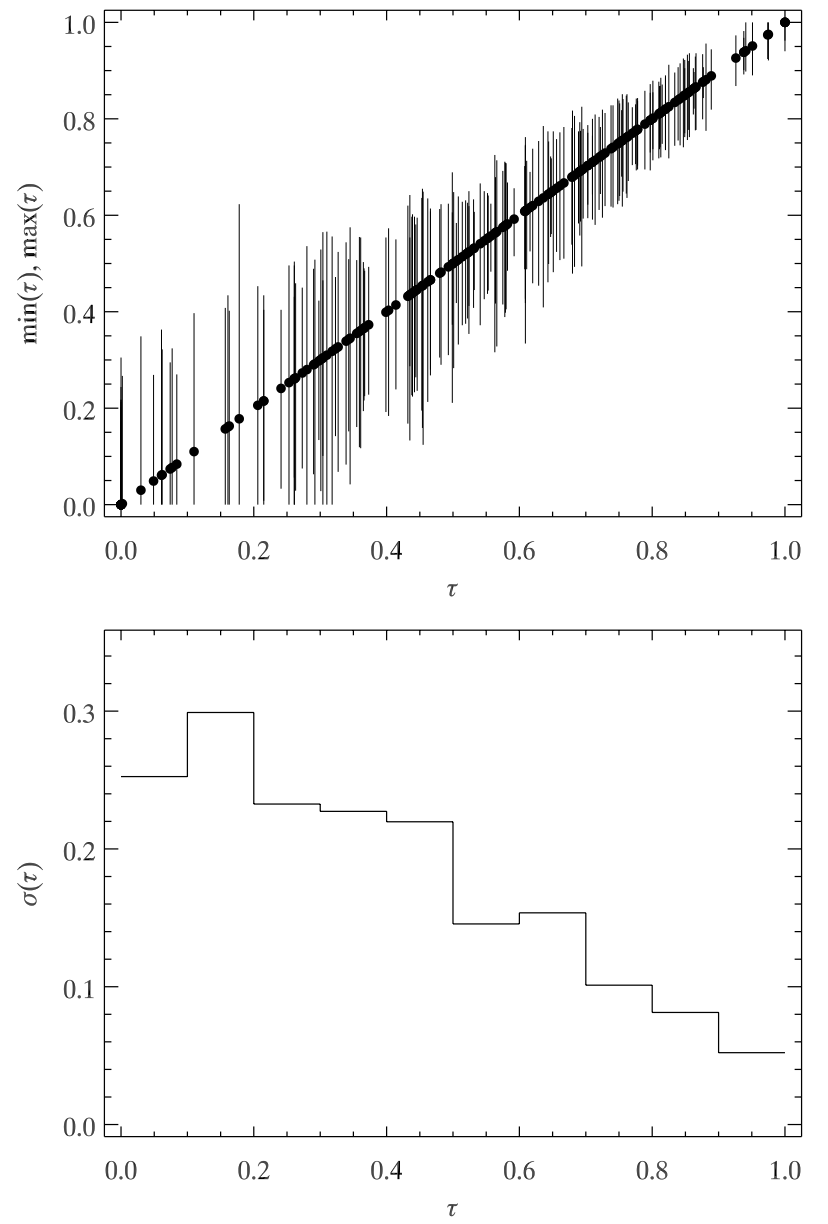

Fig. 4. Distribution of $1 \sigma$ confidence intervals of the fractional ages (top) and respective mean error bar (bottom) as a function of the relative stellar age for the studied sample of magnetic stars.

concentration of stars in the middle of the H-R diagram is seldom realised in a random sample of low mass stars drawn from a homogeneous age distribution. Denoting the corresponding probability with $P_{4}$, we have obtained $P_{4}=0.004 \%$ and $P_{4}=0.6 \%$ for stars with $M \leq 2 M_{\odot}$ and $2 M_{\odot}<M \leq 3 M_{\odot}$, respectively. At the same time, for stars with $M>3 M_{\odot}$ we have determined $P_{4}=39 \%$.

The conclusion of this series of tests is that it is very unlikely that the picture we have found is due to random errors in the determination of the stellar fundamental parameters. Unless the methods employed to measure stellar temperature and luminosity are affected by some large systematic errors, and assuming that the evolutionary models are correct, the following scenario emerges from our study. Magnetic stars with $M \leq 3 M_{\odot}$ are concentrated in the centre of the H-R diagram, and, in this mass range, older stars are more numerous than younger magnetic stars. In particular, the age distribution of stars with $M \leq 2 M_{\odot}$ might even be explained by a parent population entirely older that $\tau \approx 0.3$, scattered by random errors, whereas a similar interpretation is unlikely for stars with $2 M_{\odot}<M \leq 3 M_{\odot}$. In the latter mass range, young magnetic stars are found more rarely than expected from a distribution that is homogeneous in age, but they do exist. There are also strong indications of a lack of lower mass stars in the 

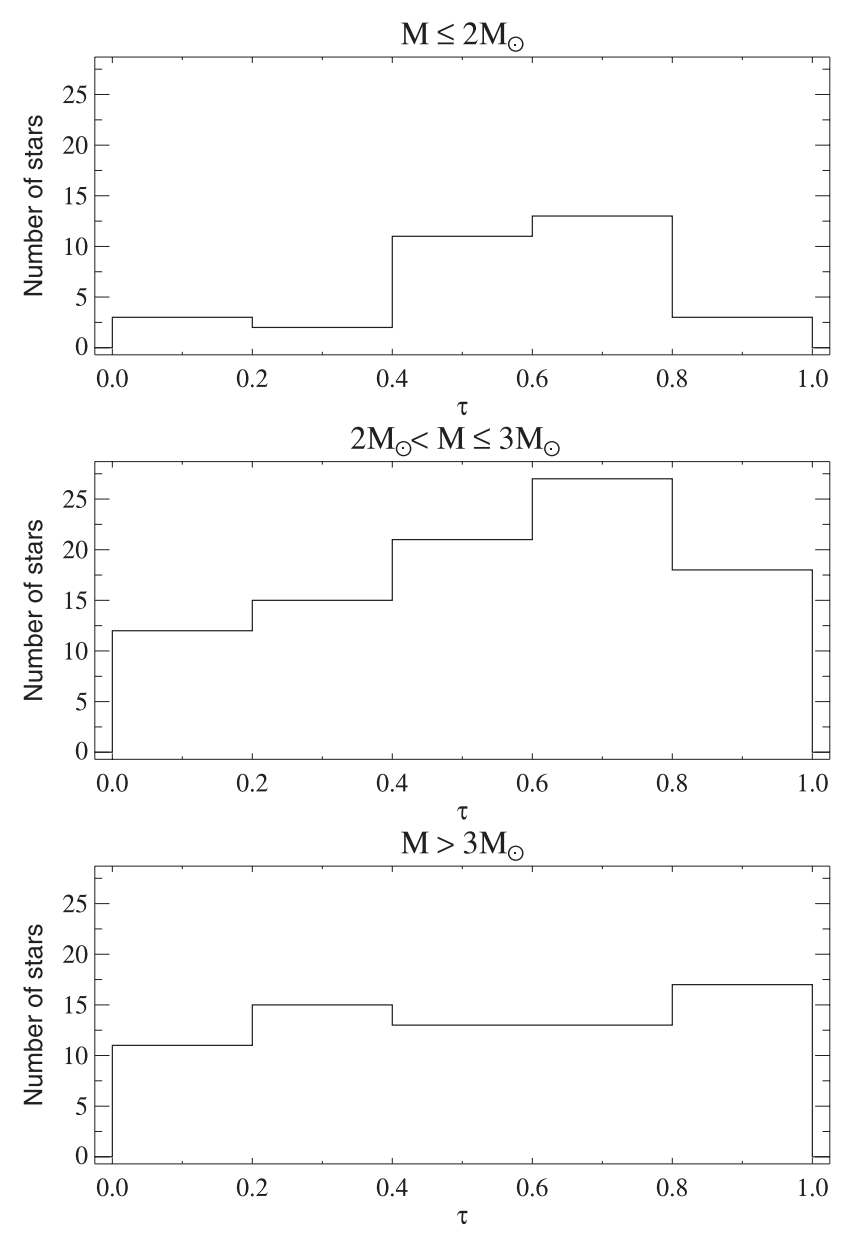

Fig. 5. Distribution of the relative ages for magnetic CP stars of different mass.

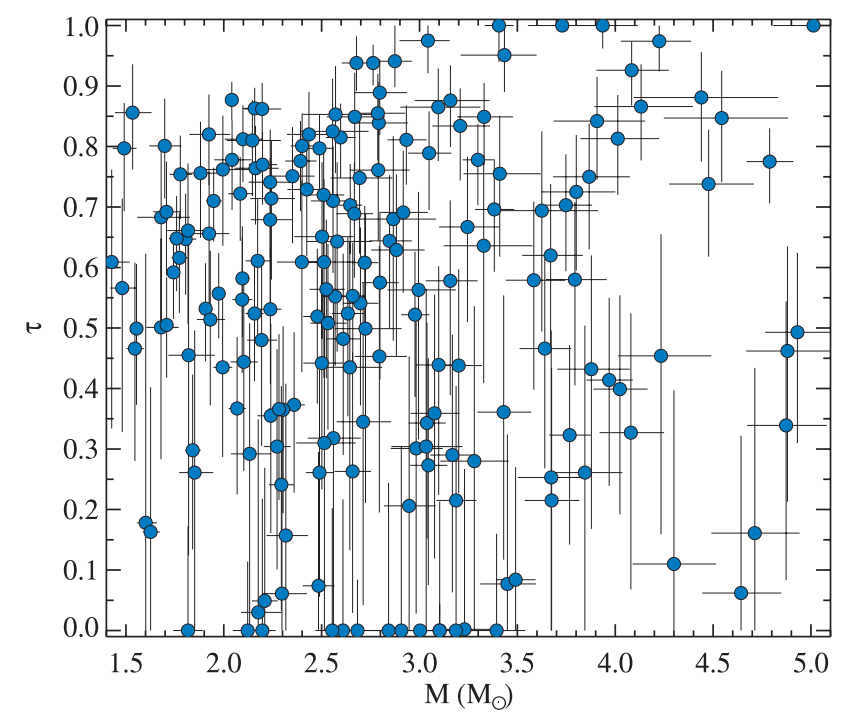

Fig. 6. Distribution of magnetic CP stars in the $\tau-M$ plane.

final stages of the main sequence evolution. Finally, magnetic CP stars with $M>3 M_{\odot}$ are homogeneously distributed in age.

Detection of the Zeeman resolved split lines was reported for 26 stars included in our sample. They all have masses below $3 M_{\odot}$ and constitute only $13 \%$ of the whole sample, which should be compared with nearly $70 \%$ of such objects in the

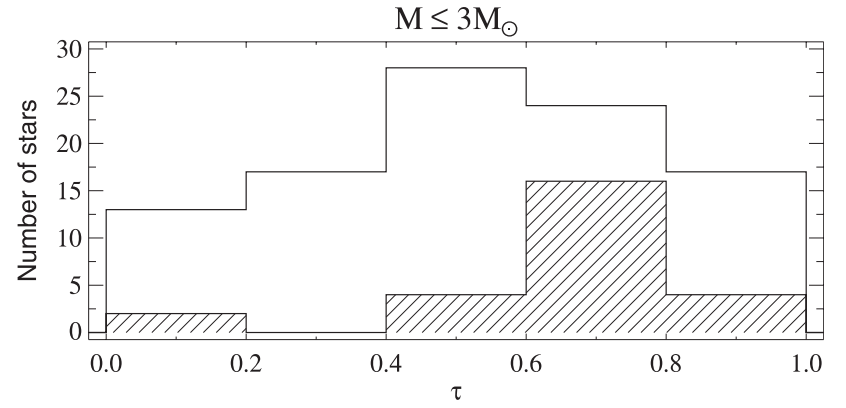

Fig. 7. Distribution of the relative ages for magnetic CP stars in the $M \leq 3 M_{\odot}$ mass group. The hatched histogram shows the age distribution for stars with the resolved Zeeman split lines, whereas the other histogram corresponds to stars without detectable Zeeman splitting.

sample analysed by Hubrig et al. (2000). In Fig. 7 we compare the age distributions of magnetic stars with and without magnetically split lines. It is clear that stars with the Zeeman resolved lines are more evolved and may not be representative of the parent population of magnetic CP stars.

\subsection{Magnetic field}

Homogeneous determination of the H-R diagram position for the large sample of magnetic CP stars allows us to investigate evolutionary changes of the surface magnetic field strength and to probe its possible dependence on the fundamental stellar parameters. We have considered the average quadratic longitudinal field (Borra et al. 1983) defined with the equation

$\left\langle\overline{B_{z}}\right\rangle=\left(\frac{1}{N} \sum_{i=1}^{N}\left\langle B_{z}\right\rangle_{i}^{2}\right)^{1 / 2}$

as magnetic field strength estimator. When available, the $\left\langle\overline{B_{z}}\right\rangle$ estimates were taken from the catalogue by Bychkov et al. (2003), otherwise we have computed $\left\langle\overline{B_{z}}\right\rangle$ from the individual longitudinal field measurements of the newly detected magnetic CP stars. For a few stars magnetic field was only observed using Zeeman resolved lines in the intensity spectra. In this case we have used approximate relation $\left\langle\overline{B_{z}}\right\rangle \approx\left\langle B_{\mathrm{s}}\right\rangle / 3$ to bring these field modulus magnetic measurements on the same scale with the average longitudinal field estimates. In addition to the observed magnetic field strength, we have computed the quantity $3\left\langle\overline{B_{z}}\right\rangle R^{2}$, which is proportional to the unsigned magnetic flux and hence provides a possibility to distinguish intrinsic evolutionary changes of the magnetic field intensity from the secular variation of the surface field strength caused by increase in the stellar radii.

The average longitudinal field and magnetic flux as a function of the relative stellar age $\tau$ are presented in Figs. 8a and b, where different panels correspond to the three mass bins defined above. Dependence of the magnetic quantities on the stellar rotation period and mass are shown in Figs. 9 and 10, respectively. The non-parametric Spearman's rank correlation coefficient $r$ (Press et al. 1992) and the associated significance $D$ were used to quantify dependencies of the magnetic quantities on the stellar parameters. 

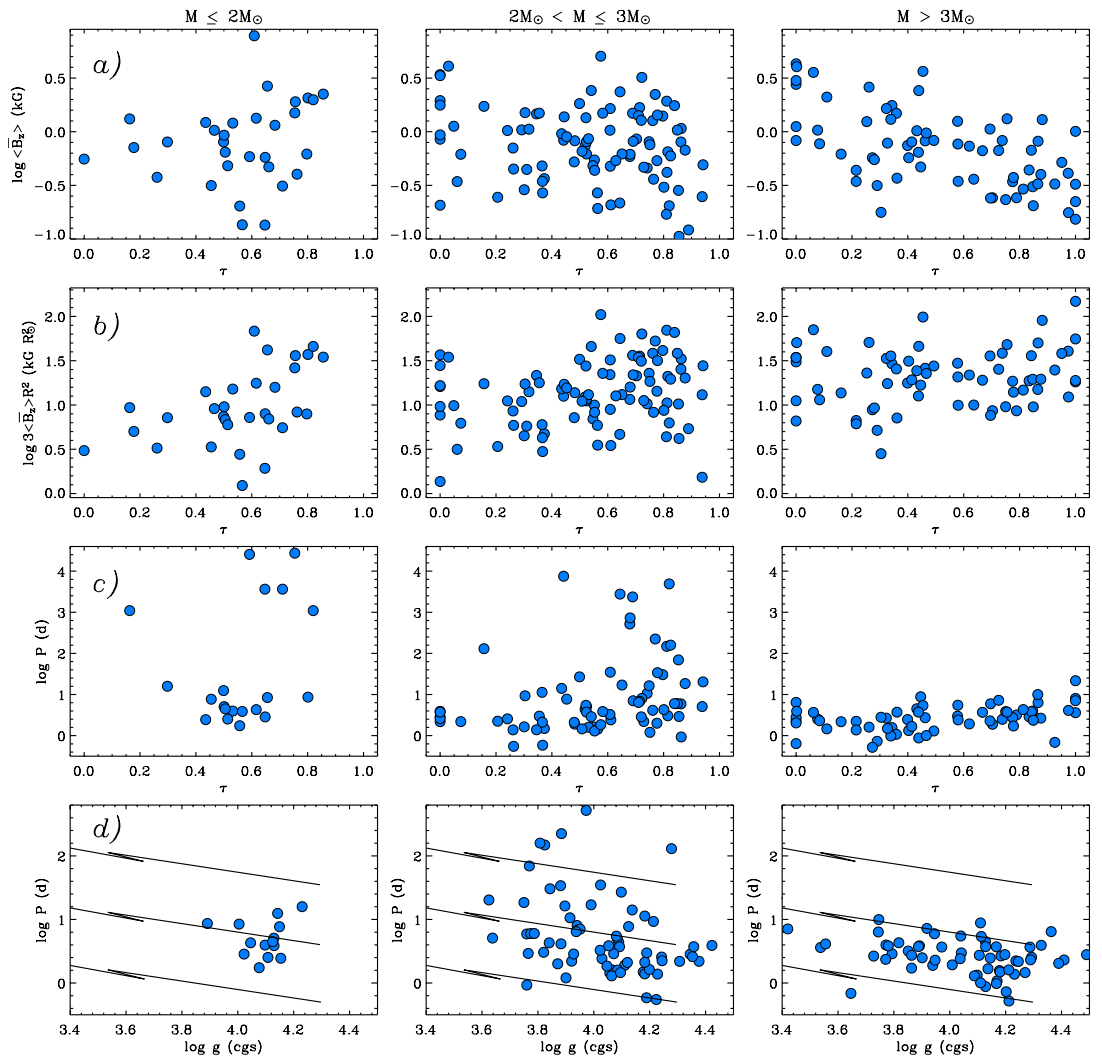

Fig. 8. The average longitudinal magnetic field (a)), magnetic flux (b)), and rotation period (c)) as a function of the relative age for magnetic CP stars with $M \leq 2 M_{\odot}$ (left column), $2 M_{\odot}<M \leq 3 M_{\odot}$ (middle column), and $M>3 M_{\odot}$ (right column). The bottom panels (d)) show rotation period as a function of surface gravity. The solid lines represent evolution of the rotation period (for initial periods of $0.5,4$, and 35 days) expected for the situation when the angular momentum is conserved.
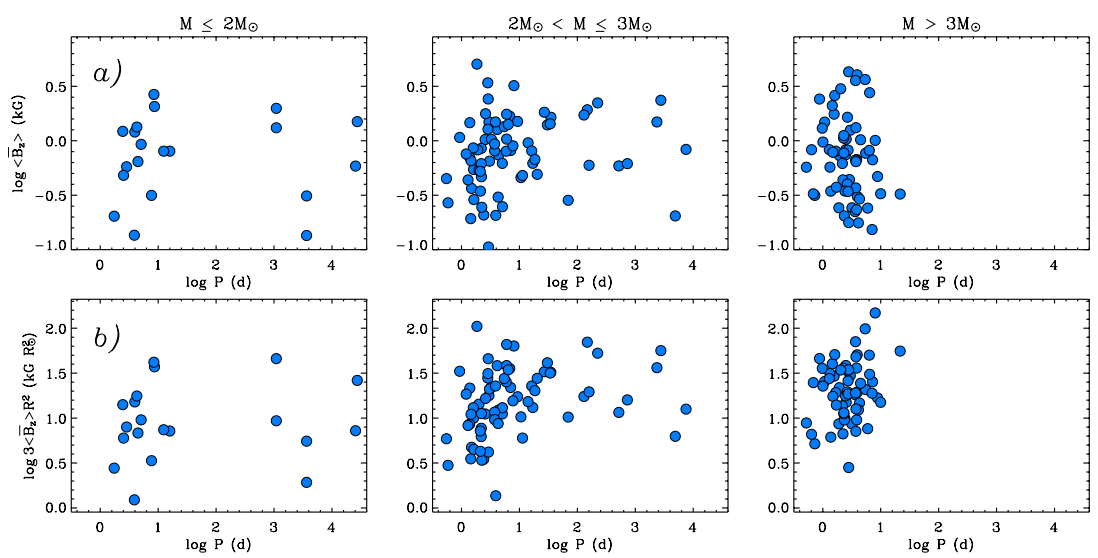

Fig. 9. The average longitudinal magnetic field (a)) and magnetic flux (b)) as a function of rotation period.

The plot of $\left\langle\overline{B_{z}}\right\rangle$ against elapsed fraction of the main sequence life reveals a significant anticorrelation $(r=-0.29$, $D>99.9 \%$ ) for the whole stellar sample and for the individual groups of stars, except for the low mass $\left(M \leq 2 M_{\odot}\right)$ objects. However, when the strong evolutionary increase of the stellar radii is accounted for, significant positive correlation ( $r=0.24$, $D>99.9 \%$ ) between the magnetic flux and $\tau$ becomes evident. This trend is significant in the group of $M \leq 3 M_{\odot}$ stars and marginal for stars with $M>3 M_{\odot}$. On the basis of this analysis we can draw the conclusion that the magnetic flux in the surface layers of low mass $\left(M \leq 3 M_{\odot}\right)$ CP stars increases with time. On average, the magnetic flux grows by almost a factor of 4 between $\tau=0$ and $\tau=1$. This effect is far less prominent (flux increase of about $40 \%$ at the $D=75 \%$ significance level) in the magnetic stars with $M>3 M_{\odot}$.

No trend of the average longitudinal field with the stellar mass is present in our data (Fig. 10). At the same time, unambiguous correlation ( $r=0.35, D>99.9 \%$ ) emerges if we consider the magnetic flux as a function of mass. We have determined the average magnetic fluxes $15.2 \pm 0.5,19.3 \pm 0.2$, and $27.2 \pm 0.3$ (in the $4 \pi \mathrm{kG} R_{\odot}^{2}$ units) for the $M \leq 2 M_{\odot}$, $2 M_{\odot}<M \leq 3 M_{\odot}$, and $M>3 M_{\odot}$ mass ranges, respectively. Thus, our investigation leads to the conclusion that massive stars are intrinsically more magnetic compared to low mass CP objects.

Information on the rotation periods of the magnetic stars in our sample was obtained from the catalogue of Catalano \& Renson (1998) and was further supplemented with the period measurements reported in several recent studies (Paunzen \& Maitzen 1998; Koen \& Eyer 2002; Ryabchikova et al. 2005). Estimates of rotation periods could be found for $80 \%$ of stars from our sample. A relation between the average longitudinal field and stellar rotation (see Fig. 9) reveals a marginal $(D=86 \%)$ correlation, which is the strongest $(D=98 \%)$ in the 

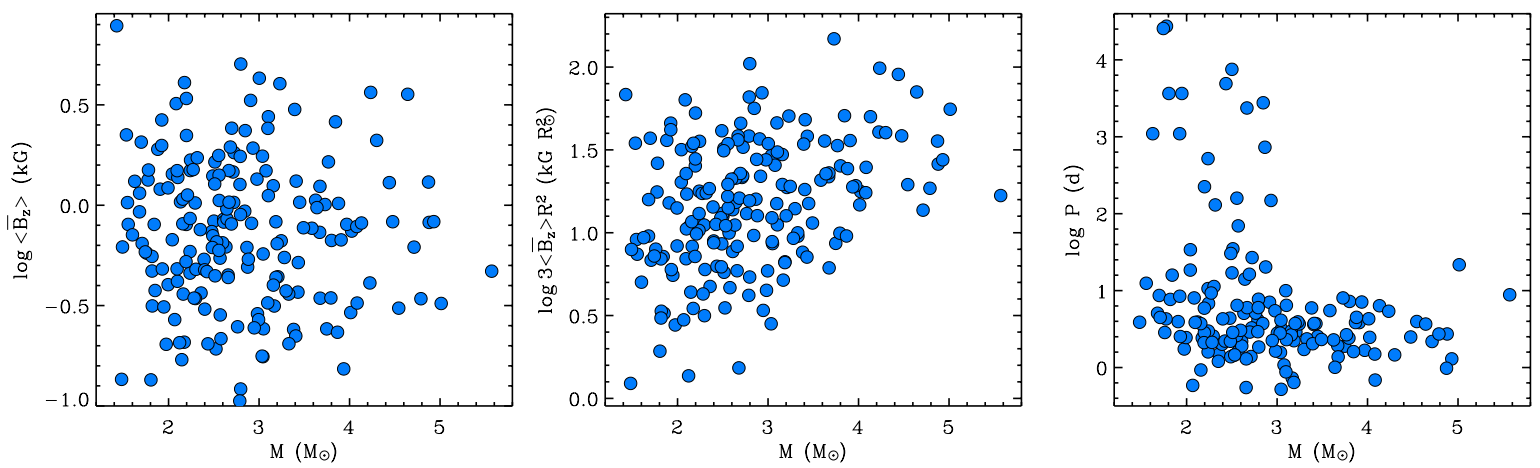

Fig. 10. The average longitudinal magnetic field (left panel), magnetic flux (middle panel), and rotation period (right panel) as a function of stellar mass.

group of stars with $2 M_{\odot}<M \leq 3 M_{\odot}$. This trend is reinforced if the magnetic flux is considered instead of the average longitudinal field. In this case, we have found $r=0.19, D=98 \%$ for the whole sample and a definite correlation $(D>99.9 \%)$ for stars in the intermediate mass range. These results indicate that the surface field is more intense in slowly rotating magnetic CP stars.

\subsection{Stellar rotation}

We have investigated evolutionary changes of the stellar rotation periods and studied a relation between rotation and fundamental stellar parameters. Rotation period as a function of the relative age, surface gravity, and stellar mass is presented in Figs. 8c,d, and 10, respectively. We have found a clear evidence that older stars have longer rotation periods. This trend is significant $(r=0.37, D>99.9 \%)$ for the whole sample and for the two groups of stars with $M>2 M_{\odot}(D>99.8 \%)$. A marginal correlation $(D=80 \%)$ is also found for the low mass objects $\left(M \leq 2 M_{\odot}\right)$.

A prominent dependence of rotation period on mass is revealed by our statistical analysis (see Fig. 10). Rotation periods of the most massive $\left(M>3 M_{\odot}\right)$ magnetic CP stars show a fairly sharp cutoff at $P_{\text {rot }} \approx 10$ days. In contrast, there are many instances of much slower rotation among less massive $\left(M \leq 3 M_{\odot}\right)$ CP stars.

Possible changes of the total angular momentum and secular evolution of the stellar moment of inertia can both contribute to the observed variation of rotation periods. In order to isolate a signature of the angular momentum evolution, we have followed North (1998) and studied rotation period as a function of surface gravity. In Fig. 8d the observed relation is compared with the changes of rotation period predicted for rigidly rotating stars that conserve the total angular momentum during their life at the main sequence. These theoretical curves, adopted from North (1998), are virtually mass independent and are plotted in Fig. 8d for initial periods of 0.5, 4, and 35 days. From this comparison we have found that the observed periodage dependence of stars with $M>3 M_{\odot}$ is fully accounted for by the changes of the moment of inertia. Thus, we see no indication of significant changes in the angular momentum of the most massive magnetic CP stars. At the same time, the group of stars in the $2 M_{\odot}<M \leq 3 M_{\odot}$ mass range shows an abnormally steep slope in their $\log P_{\text {rot }}-\log g$ diagram, suggesting that these stars may have experienced some loss of the angular momentum during the main sequence evolutionary stage. If we subtract theoretically expected period trend from our data, a marginal residual anticorrelation $(r=-0.13$, $D=71 \%$ ) of rotation period with respect to $\log g$ is still present. The large scatter of data points precludes us from quantifying the angular momentum evolution history of the low mass magnetic CP stars in more detail.

\section{Discussion and conclusions}

We have carried out a detailed statistical investigation of the evolutionary state of the upper main sequence magnetic CP stars. The sample of 194 objects has included all CP stars whose magnetic status could be confirmed with a direct detection of the surface magnetic field and for which precise parallax is available in the Hipparcos catalogue. The literature data on the magnetic observations of $\mathrm{CP}$ stars were complemented with the analysis of the archival spectropolarimetric data acquired with the FORS1 instrument at ESO VLT. This allowed us to detect magnetic field in $53 \mathrm{~A}$ and B-type CP stars, of which only five were previously known to be magnetic. Using the medium-band (Geneva or Strömgren) photometry of the program stars, we have determined $T_{\text {eff }}$ and then have placed stars on the H-R diagram. We were able to obtain stellar masses, radii, and ages from the comparison of the observed luminosity and temperature with the predictions of the theoretical evolutionary tracks. Our investigation has included a comprehensive non-linear error analysis, that has permitted us to quantify uncertainty of the derived stellar properties for each period of the main sequence life. Numerical simulations were applied to assess the properties of the resulting age distributions of the magnetic CP stars of different masses. We have also employed correlation analysis to study dependence of the surface magnetic field, magnetic flux, and rotation period on the stellar mass and to probe possible evolutionary changes of the stellar rotation and magnetic field.

The key results of our study can be summarised as follows.

1. The most massive magnetic CP stars $\left(M>3 M_{\odot}\right)$ are distributed homogeneously in the main sequence band. On the other hand, stars with $M \leq 3 M_{\odot}$ show the tendency to cluster in the middle of the main sequence. The relative 
shortage of young and very old stars is especially pronounced in the group of low mass $\left(M \leq 2 M_{\odot}\right)$ stars. This uneven age distribution cannot be attributed to the effect of random errors in determination of the stellar parameters.

2. We have found 22 young $(\tau \leq 0.3)$ magnetic stars among the objects with $M \leq 3 M_{\odot}$, thereby rejecting the proposal by Hubrig et al. (2000) that all observably magnetic low mass CP stars have completed significant fraction of their main sequence evolution. At the same time, our data for the least massive $\left(M \leq 2 M_{\odot}\right)$ stars is not inconsistent with a population of stars older than $\tau=0.3$.

3. The average surface field observed in magnetic CP stars decreases with time. However, for stars with $M \leq 3 M_{\odot}$ this decrease is slower than the field weakening computed under the assumption of the magnetic flux conservation. Consequently, we suggest that the surface magnetic flux of the low mass CP stars increases with time.

4. Comparison of the average magnetic fluxes of the CP stars from different mass ranges shows that massive $\mathrm{CP}$ stars are substantially more magnetic. At the same time, we found a correlation between magnetic field and rotation period for the intermediate mass stars.

5. Rotation period of magnetic stars increases with time, but for stars with $M>3 M_{\odot}$ the stellar structure changes influencing the stellar moment of inertia can fully account for the observed period increase. On the other hand, our results do not rule out the possibility that angular momentum losses occur during the main sequence evolution of stars with $2 M_{\odot}<M \leq 3 M_{\odot}$.

The conspicuous inhomogeneous age distribution of the low mass magnetic $\mathrm{CP}$ stars which has emerged from our statistical analysis requires careful verification and interpretation. Our results raise the question whether the anomalous H-R diagram distribution is the property of stars with significant surface magnetic field or it is intrinsic to the whole group of the $\mathrm{SrCrEu}$ and less massive Si-type stars. No significant anomalies in the evolutionary state of these subclasses of CP stars were reported by Gomez et al. (1998). However, the adopted upper threshold of the relative parallax uncertainty and the methods used in the Gomez et al. study differ substantially from the analysis procedure employed in the present investigation. Methodologically more similar analysis by Pöhnl et al. (2005) included only 15 stars with $M \leq 2 M_{\odot}$, and most of these objects were found in the centre of the main sequence band, very similar to our results.

An indirect hint that the $\mathrm{SrCrEu}$ and $\mathrm{Si}$ chemical peculiarity is closely related to the presence of magnetic field at the stellar surface comes from the survey of bright Ap stars carried out by Aurière et al. (2004). Using sensitive spectropolarimetric field diagnostic methods, these authors were able to detect magnetic field in essentially every peculiar star they have observed and have established a possible lower threshold of $\approx 250 \mathrm{G}$ for the strength of the dipolar field component. In the light of these findings and taking results of our study into account, an investigation of the evolutionary status of all $\mathrm{SrCrEu}$ and $\mathrm{Si}$ stars with $M \leq 3 M_{\odot}$ would be of great importance and could benefit from the upcoming revision of the Hipparcos data reduction (van Leeuwen \& Fantino 2005).

For many decades the problem of the evolution of global magnetic field in A and B stars was approached in the framework of the analysis of the Ohmic decay of the poloidal fossil field, assumed to exist in stellar interior (Cowling 1945; Moss 1984; Landstreet 1987). Analytical estimates predict appreciable field decay only after $\sim 10^{10}-10^{11} \mathrm{yr}$, which exceeds the main sequence lifetime of even the least massive magnetic CP stars. Consequently, any observation of the possible secular evolution of magnetic field was often considered as a challenge for the fossil field theory. However, this classical assessment may be fundamentally flawed due to neglect of the toroidal field component, which must exist in the interior of magnetic CP stars in order to ensure dynamical stability of their global fields (Prendergast 1956; Tayler 1980). Recent numerical MHD simulations of the fossil field dynamics in radiative stellar interiors by Braithwaite \& Spruit (2004, see also Braithwaite \& Nordlund 2006) have established that instability of the poloidal field component can be suppressed by the presence of the interior toroidal magnetic component of similar strength. The diffusive evolution of such twisted fields in the simulations by Braithwaite \& Nordlund (2006) is determined essentially by the toroidal field. In their model the total magnetic energy decreases with time, but the surface field strength is expected to increase, until the toroidal component emerges at the surface and the field decays rapidly. The time scale of this process is $\sim 2 \times 10^{9} \mathrm{yr}$ for a $2 M_{\odot}$ star (Braithwaite \& Nordlund 2006), but the precise value is rather uncertain due to a very schematic treatment of the atmospheric magnetic reconnection and because of the sensitivity to the assumed initial field structure. These difficulties notwithstanding, the recent numerical work has emphasized limitations of the traditional analytical studies of the global field evolution and suggested that an observation of the long-term systematic change of the surface field is not necessarily incompatible with the fossil field hypothesis. Our observation of the uneven distribution of the low mass magnetic CP stars in the H-R diagram and of the possible increase in their surface magnetic flux with time may be plausibly interpreted as a signature of the Ohmic diffusion of the twisted fossil field. The relative shortage of the old low mass stars may indicate that in these objects we are observing the final stage of the field emergence and rapid decay. If this scenario is correct, the field starts to emerge in stars with $M \lesssim 2 M_{\odot}$ after $\sim 4 \times 10^{8}$ yr of the main sequence evolution and completes its decay after $\sim 10^{9} \mathrm{yr}$.

The difference in the age distribution and magnetic field properties of the low and high mass $\mathrm{CP}$ stars may be related to their pre-main sequence (PMS) evolutionary history and, especially, to the behaviour of the interior and envelope convective zones. One can argue (e.g., Tout et al. 2004) that a large-scale coherent field typical of magnetic CP stars can be frozen in, or undergo a slow diffusive evolution as envisaged by Braithwaite $\&$ Nordlund (2006), in the fully radiative parts of the stellar interiors. In contrast, the turbulence in the convective zones leads to rapid reconnection of the field lines and thus contributes to the dissipation of the magnetic flux. Consequently, primordial field can survive only in stars that do not pass through a long 
fully convective phase during their approach to the ZAMS and in the subsequent main sequence evolution. Theoretical calculations of the PMS stellar evolution by Palla \& Stahler (1993) have shown that, although the low mass stars are fully convective during a large fraction of their PMS life, the duration of this phase decreases rapidly with an increasing stellar mass. A $1.5 M_{\odot}$ star is expected to spend roughly $10^{5}$ years in the fully convective phase, which is already a factor of ten shorter compared to a solar-type star and is probably too short to destroy the fossil magnetic field completely. For masses of $\gtrsim 2.4 M_{\odot}$ the PMS star is never fully convective, which facilitates survival of the primordial field. Thus, the observed difference in the age distribution of the low and high mass magnetic CP stars may reflect the history of the field dissipation in the convectively unstable regions of stellar interior. The presence of more extended and long-lived PMS convective zones in stars with $M \lesssim 2.4-2.0 M_{\odot}$ suggests that these objects are more likely to possess weak or no field in the outer regions when they reach the ZAMS. Subsequent increase in the field strength results from the outward expansion of magnetic field into the newly formed radiative regions. In contrast to this scenario of the field behaviour in the low mass stars, the fossil field in more massive magnetic CP stars is probably not significantly altered by the feeble PMS convective zones and appears close to the stellar surface even in young stars.

In the context of the study of the origin and evolution of the magnetic field in CP stars it is helpful also to investigate the evolution of the stellar angular momentum. Magnetic $\mathrm{CP}$ stars have generally longer rotation periods than normal A and B stars. The bulk of their rotation rates forms a separate Maxwellian distribution with an average value 3-4 times lower than that found in normal A and B-type stars (Stępień 2000), but there are also groups of $\mathrm{CP}$ stars with rotation period of years (Mathys et al. 1997) or decades (e.g., $\gamma$ Equ, see Leroy et al. 1994). Several works suggest that neither field CP stars nor cluster CP stars undergo any significant magnetic braking during their life on the main sequence (North 1998; this study). Therefore, angular momentum must be lost before the star reaches the ZAMS. Stępień (2000) explains the slow rotation as the result of an interaction of the stellar magnetic field with the circumstellar environment during the PMS phase. And if a magnetised wind still persists after the dissipation of the circumstellar disk, a PMS CP star may further slow down, reaching the ZAMS with an extremely long rotation period. In this respect, the very existence of slow rotating stars with strong magnetic fields suggests that magnetic field was already present when the stars were in the pre-main sequence phase. For stars with $M>2 M_{\odot}$ this hypothesis is supported by the discovery of a magnetic field in NGC 2244334 , a $4 M_{\odot}$ star that has spent only $2 \%$ of its life in the main sequence (Bagnulo et al. 2004), and in HD 66318, a star with a mass of $2.1 M_{\odot}$ and $\tau=0.16$, that belongs to the open cluster NGC 2516. More direct confirmation that magnetic fields are present during the pre-main sequence phase comes from the discovery of magnetic field in Herbig Ae/Be stars (considered the progenitors of main sequence early-type stars) by Wade et al. (2005).

The most puzzling evolutionary properties are observed for the group of stars with $M \leq 2 M_{\odot}$, which contains very few young stars and no stars approaching the terminal age main sequence. Observational evidence for lack of young lower mass $\left(M \lesssim 2 M_{\odot}\right)$ Ap stars comes also from open cluster studies. Abt (1979) suggested that low-mass Ap stars are found only in clusters that are at least $10^{8} \mathrm{yr}$ old, and a study of four nearby young clusters by Pöhnl et al. (2003) is also consistent with this finding. Assuming that magnetic breaking during pre-main sequence phase is the reason why these stars rotate slower than normal late A-type stars, we face a scenario in which magnetic field appears and disappears from the stellar surface several times during the star's life. Magnetic field was present at the surface of the star during the pre-main sequence phase, then disappeared when the star reached the ZAMS, to appear again at a more evolved state, and disappear toward the end of the star's life in the main sequence. At the same time, one can argue that magnetic field responsible for the PMS angular momentum loss is not directly related to the fossil field seen at the surfaces of main sequence low mass magnetic CP stars. The former field may be generated by the dynamo processes or represent the outer part of the fossil field tangled by the envelope convection. This complex field decays by the time the star reaches the ZAMS and, after some evolution on the main sequence, the interior fossil field which has retained its global organisation appears at the surface.

Our study is the first investigation which included enough very low mass magnetic $\mathrm{CP}$ stars to identify interesting characteristics of this stellar group, yet it is clear that the number of stars in the corresponding mass range is still rather small. This circumstance does not permit us to draw definite conclusions about the nature of the anomalies in the age distribution observed for these stars. Thus, we call for a more detailed analysis of the rotation, magnetic, and evolutionary characteristics of CP stars with $M \leq 2 M_{\odot}$. Such an investigation would represent the most interesting follow up of the present study.

Acknowledgements. This research has made extensive use of the SIMBAD database, operated at CDS, Strasbourg, France, and NASA's Astrophysics Data System Bibliographic Services. OK acknowledges funding from the Scientific Visitors Programme of ESO Chile. We acknowledge the use of ESO Science Archive Facility.

\section{References}

Abt, H. A. 1979, ApJ, 230, 485

Aurière, M., Silvester, J., Wade, G. A., et al. 2004, in The A-star puzzle, ed. J. Zverko, J. Žižňovský, S. J. Adelman, \& W. W. Weiss (Cambridge University Press), IAU Symp., 224, 633

Aznar Cuadrado, R., Jordan, S., Napiwotzki, R., et al. 2004, A\&A, 423, 1081

Babcock, H. W. 1958, ApJS, 3, 141

Bagnulo, S., Szeifert, T., Wade, G. A., Landstreet, J. D., \& Mathys, G. 2002, A\&A, 389, 191

Bagnulo, S., Landstreet, J. D., Lo Curto, G., Szeifert, T., \& Wade, G. A. 2003, A\&A, 403, 645

Bagnulo, S., Hensberge, H., Landstreet, J. D., Szeifert, T., \& Wade, G. A. 2004, A\&A, 416, 1149

Bagnulo, S., Landstreet, J. D., Mason, E., et al. 2005, A\&A, submitted Bessell, M. 2000, in Encyclopedia of Astronomy and Astrophysics (IOP Publishing Ltd and Nature Publishing Group), 1638

Borra, E. F., \& Landstreet, J. D. 1980, ApJS, 42, 421 
Borra, E. F., \& Landstreet, J. D., \& Thompson, I. 1983, ApJS, 53, 151 Braithwaite, J., \& Spruit, H. C. 2004, Nature, 431, 819

Braithwaite, J., \& Nordlund, А̊ 2006, A\&A, in press [arXiv: astro-ph/0510316]

Bychkov, V. D., Bychkova, L. V., \& Madej, J. 2003, A\&A, 407, 631

Casini, R., \& Landi Degl'Innocenti, E. 1994, A\&A, 291, 668

Catalano, F. A., \& Renson, P. 1998, A\&AS, 127, 421

Cowley, C. R., Ryabchikova, T., Kupka, F., et al. 2000, MNRAS, 317, 299

Cramer, N. 1982, A\&A, 112, 330

Cowling, T. G. 1945, MNRAS, 105, 166

El'kin, V. G., Kudryavtsev, D. O., \& Romanyuk, I. I. 2003, Astron. Lett., 29, 400

Fitzpatrick, E. L. 1999, PASP, 111, 63

Flower, P. J. 1996, ApJ, 469, 355

Johnson, N. M. 2004, M. Sc. thesis, Royal Military College of Canada Jordan, S., Werner, K., \& O'Toole, S. J. 2005, A\&A, 432, 273

Golay, M. 1972, Vistas in Astron., 14, 13

Gomez, A. E., Luri, X., Grenier, S., et al. 1998, A\&A, 336, 953

Hakkila, J., Myers, J. M., Stidham, B. J., \& Hartmann, D. H. 1997, AJ, 114, 2043

Hauck, B., \& North, P. 1993, A\&A, 269, 403

Hauck, B., \& Künzli, M. 1996, Baltic Astron., 5, 303

Hauck, B., \& Mermilliod, M. 1998, A\&AS, 129, 431

Hubrig, S., \& Castelli, F. 2001, A\&A, 375, 963

Hubrig, S., North, P., \& Mathys, G. 2000, ApJ, 539, 352

Hubrig, S., Szeifert, T., Schöller, M., Mathys, G., \& Kurtz, D. W. 2004, A\&A, 415, 685

Hubrig, S., Nesvacil, N., Schöller, M., et al. 2005, A\&A, 2005, A\&A, 440, L37

Kochukhov, O., Bagnulo, S., \& Barklem, P. S. 2002, ApJ, 578, L75

Koen, C., \& Eyer, L. 2002, MNRAS, 331, 45

Künzli, M., North, P., Kurucz, R. L., \& Nicolet, B. 1997, A\&AS, 122, 51

Landstreet, J. D. 1987, MNRAS, 225, 437

Lanz, T. 1984, A\&A, 139, 161

Leckrone, D. S. 1973, ApJ, 185, 577

Leroy, J. L., Bagnulo, S., Landolfi, M., \& Landi Degl'Innocenti, E. 1994, A\&A, 284, 174

Lucke, P. B. 1978, A\&A, 64, 367

Lutz, T. E, \& Kelker, D. H. 1973, PASP, 85, 573

Mathys, G. 1991, A\&AS, 89, 121

Mathys, G., \& Lanz, T. 1990, A\&A, 230, L21

Mathys, G., Hubrig, S., Landstreet, J. D., Lanz, T., \& Manfroid, J. 1997, A\&AS, 123, 353

Moon, T. T., \& Dworetsky, M. M. 1985, MNRAS, 217, 305

Moss, D. 1984, MNRAS, 207, 107

Moss, D. 1989, MNRAS, 236, 629
Moss, D. 2004, in The A-star puzzle, ed. J. Zverko, J. Žižňovský, S. J. Adelman, \& W. W. Weiss (Cambridge University Press), IAU Symp., 224, 245

North, P. 1981, A\&A, 97, 359

North, P. 1998, A\&A, 334, 181

O’Toole, S. J., Jordan, S., Friedrich, S., \& Heber, U. 2005, A\&A, 437, 227

Palla, F., \& Stahler, S. W. 1993, ApJ, 418, 414

Paunzen, E., \& Maitzen, H. M. 1998, A\&AS, 133, 1

Perryman, M. A. C., Lindegren, L., Kovalevsky, J., et al. 1997, A\&AS, 323,49

Pöhnl, H., Maitzen, H. M., \& Paunzen, E. 2003, A\&A, 402, 247

Pöhnl, H., Paunzen, E., \& Maitzen, H. M. 2005, A\&A, 441, 1111

Prendergast, K. H. 1956, ApJ, 123, 498

Press, W. H., Teukolsky, S. A., Vetterling, W. T., \& Flannery, B. P. 1992, Numerical Recipes in FORTRAN. The Art of Scientific Computing, 2nd edn. (Cambridge: Cambridge Univ. Press)

Renson, P., Gerbaldi, M., \& Catalano, F. A. 1991, A\&AS, 89, 429

Romanyuk, I. I. 2000, in Magnetic Fields of Chemically Peculiar and Related Stars, ed. Yu. V. Glagolevskij, \& I. I. Romanyuk, Moscow, 18

Ryabchikova, T., Leone, F., Kochukhov, O., \& Bagnulo, S. 2004, in The A-star puzzle, ed. J. Zverko, J. Žižňovský, S. J. Adelman, \& W. W. Weiss (Cambridge University Press), IAU Symp., 224, 580

Ryabchikova, T., Wade, G. A., Aurière, M., et al. 2005, A\&A, 429, L55

Rufener, F. 1989, A\&AS, 78, 469

Schaller, G., Schaerer, D., Meynet, G., \& Maeder, A. 1992, A\&AS, 96, 269

Schaerer, D., Charbonnel, C., Meynet, G., Maeder, A., \& Schaller, G. 1993, A\&AS, 102, 339

Schlegel, D. J., Finkbeiner, D. P., \& Davis, M. 1998, ApJ, 500, 525

Shorlin, S. L. S., Wade, G. A., Donati, J.-F., et al. 2002, A\&A, 392, 637

Smith, H. J. 2003, MNRAS, 338, 891

Sofia, U. J., \& Meyer, D. M. 2001, ApJ, 588, L147

Stępień, K. 2000, A\&A, 353, 227

Stępień, K. 2004, in Magnetic Stars, ed. Yu. V. Glagolevskij, D. O. Kudryavtsev, \& I. I. Romanyuk, Niznij Arkhyz, 19

Stütz, Ch., Ryabchikova, T., \& Weiss, W. W. 2003, A\&A, 402, 729

Tayler, R. J. 1980, MNRAS, 191, 151

Tout, C. A., Wikramasinghe, D. T., \& Ferrario, L. 2004, MNRAS, 355 , L13

van Leeuwen, F., \& Fantino, E. 2005, A\&A, 439, 791

Wade, G. A., Aurière, M., Donati, J.-F., et al. 2003, in Modelling of Stellar Atmospheres, ed. N. E. Piskunov, W. W. Weiss, \& D. F. Gray (ASP), IAU Symp., 210, D29

Wade, G. A., Drouin, D., Bagnulo, S., et al. 2005, A\&A, 442, L31 
O. Kochukhov and S. Bagnulo: Evolutionary state of magnetic chemically peculiar stars, Online Material p 1

\section{Online Material}


Table 1. $\left\langle B_{z}\right\rangle$ measurements for the sample of CP stars observed with FORS1 (data retrieved from the ESO archive). Columns 1 and 2 list the $\mathrm{HD}$ and HIP identification. Columns 3 and 4 give the $V$-magnitude and spectral type, respectively, most of which are extracted from the General catalogue of Ap and Am stars by Renson et al. (1991). Column 5 gives the Julian Date of the middle of the exposures. Column 6 reports $\left\langle B_{z}\right\rangle$ with its error bar in Gauss. The last column indicates stars for which magnetic field was detected for the first time (new detection, ND) or confirmed (confirmed detection, CD).

\begin{tabular}{|c|c|c|c|c|c|c|}
\hline HD & HIP & $\bar{V}$ & Sp. type & JD & $\left\langle B_{z}\right\rangle(\mathrm{G})$ & Comment \\
\hline \multirow{3}{*}{1048} & 1193 & 6.2 & A1 Si & 2452910.603 & $403 \pm 105$ & ND \\
\hline & & & & 2453199.906 & $-89 \pm 46$ & \\
\hline & & & & 2453215.882 & $36 \pm 45$ & \\
\hline 3326 & 2852 & 6.1 & A6 Sr & 2452908.690 & $99 \pm 62$ & \\
\hline 8783 & 6534 & 7.8 & $\mathrm{~A} 2 \mathrm{SrCrEu}$ & 2452852.858 & $-29 \pm 106$ & \\
\hline 10840 & 8132 & 6.8 & B9 Si & 2453184.831 & $-148 \pm 81$ & \\
\hline \multirow[t]{2}{*}{19712} & 14736 & 7.3 & $\mathrm{~A} 0 \mathrm{CrEu}$ & 2452905.884 & $-930 \pm 109$ & ND \\
\hline & & & & 2452999.525 & $764 \pm 66$ & \\
\hline 19918 & 14026 & 9.4 & $\mathrm{~A} 5 \mathrm{SrCrEu}$ & 2452908.711 & $-777 \pm 109$ & ND \\
\hline \multirow[t]{2}{*}{22374} & 16859 & 6.7 & $\mathrm{~A} 1 \mathrm{CrSrSi}$ & 2452999.538 & $-10 \pm 64$ & \\
\hline & & & & 2453216.880 & $63 \pm 36$ & \\
\hline 22488 & 16527 & 7.7 & $\mathrm{~A} 3 \mathrm{SrCrEu}$ & 2453087.514 & $114 \pm 51$ & \\
\hline \multirow[t]{2}{*}{23207} & 17345 & 7.5 & $\mathrm{~A} 2 \mathrm{SrEu}$ & 2453215.861 & $259 \pm 72$ & ND \\
\hline & & & & 2453218.835 & $394 \pm 53$ & \\
\hline 23408 & 17573 & 3.9 & B7 He-weak Mn & 2452963.656 & $24 \pm 65$ & \\
\hline 24188 & 17543 & 6.3 & $\mathrm{~A} 0 \mathrm{Si}$ & 2453087.532 & $426 \pm 48$ & ND \\
\hline 30612 & 21949 & 5.5 & B9 Si & 2453087.546 & $44 \pm 44$ & \\
\hline 34797 & 24827 & 6.5 & B8 He-weak Si & 2452999.566 & $1059 \pm 86$ & ND \\
\hline 42659 & 29365 & 6.7 & $\mathrm{~A} 3 \mathrm{SrCrEu}$ & 2452999.619 & $418 \pm 78$ & ND \\
\hline \multirow[t]{2}{*}{55522} & 34798 & 5.9 & B2 Si He & 2452999.699 & $168 \pm 81$ & \\
\hline & & & & 2453000.553 & $821 \pm 68$ & ND \\
\hline 56350 & 34929 & 6.7 & A0 $\mathrm{SrCrEu}$ & 2452999.739 & $824 \pm 86$ & ND \\
\hline 56455 & 35029 & 5.7 & $\mathrm{~A} 0 \mathrm{Si}$ & 2452999.751 & $85 \pm 98$ & \\
\hline 58448 & 35676 & 7.1 & B8 Si & 2452999.765 & $60 \pm 99$ & \\
\hline 60435 & 36537 & 8.9 & $\mathrm{~A} 3 \mathrm{SrEu}$ & 2453000.572 & $-315 \pm 87$ & ND \\
\hline \multirow[t]{2}{*}{63401} & 37982 & 6.3 & B9 Si & 2453002.553 & $48 \pm 113$ & \\
\hline & & & & 2453004.728 & $-486 \pm 106$ & ND \\
\hline 74168 & 42519 & 7.5 & B9 Si & 2453002.611 & $-75 \pm 76$ & \\
\hline 74196 & 42535 & 5.6 & B7 He-weak & 2452906.888 & $121 \pm 122$ & \\
\hline \multirow[t]{2}{*}{75989} & 43528 & 6.5 & B9 $\mathrm{Si}$ & 2452992.841 & $-279 \pm 161$ & \\
\hline & & & & 2453004.783 & $-46 \pm 102$ & \\
\hline 80316 & 45658 & 7.8 & $\mathrm{~A} 3 \mathrm{SrEu}$ & 2452992.857 & $-269 \pm 134$ & \\
\hline 83625 & 47272 & 6.9 & A0 Si Sr & 2453008.822 & $-1484 \pm 83$ & ND \\
\hline 84041 & & 9.4 & $\mathrm{~A} 5 \mathrm{SrEu}$ & 2453002.670 & $497 \pm 91$ & ND \\
\hline 86181 & 48619 & 9.4 & $\mathrm{~F} 0 \mathrm{Sr}$ & 2453002.692 & $360 \pm 77$ & ND \\
\hline 86199 & 48643 & 6.7 & B9 Si & 2453003.845 & $-768 \pm 89$ & ND \\
\hline 88158 & 49642 & 6.5 & B8 Si & 2453008.838 & $233 \pm 77$ & ND \\
\hline 88385 & 49791 & 8.1 & $\mathrm{~A} 0 \mathrm{SiCrEu}$ & 2453010.681 & $-958 \pm 70$ & ND \\
\hline 89103 & 50248 & 7.8 & B9 Si & 2453010.702 & $-1949 \pm 84$ & ND \\
\hline 89385 & 50398 & 8.4 & B9 $\mathrm{SiCrEu}$ & 2453010.718 & $-81 \pm 87$ & \\
\hline 91239 & 51512 & 7.4 & B9 $\mathrm{SiCrEu}$ & 2453118.559 & $-116 \pm 77$ & \\
\hline \multirow[t]{2}{*}{92106} & 51632 & 7.8 & $\mathrm{~A} 0 \mathrm{SrCrEu}$ & 2453010.739 & $72 \pm 98$ & \\
\hline & & & & 2453118.580 & $-89 \pm 90$ & \\
\hline \multirow[t]{2}{*}{92385} & 52059 & 6.7 & B9 Si & 2453008.869 & $-588 \pm 90$ & ND \\
\hline & & & & 2453020.832 & $240 \pm 101$ & \\
\hline \multirow[t]{3}{*}{92499} & 52218 & 8.9 & $\mathrm{~A} 2 \mathrm{SrCrEu}$ & 2453010.755 & $-1163 \pm 324$ & ND \\
\hline & & & & 2453011.712 & $-1230 \pm 124$ & \\
\hline & & & & 2453118.595 & $-989 \pm 141$ & \\
\hline 93030 & 52419 & 2.7 & B0 Si N P & 2453012.731 & $-46 \pm 132$ & \\
\hline 96451 & 54166 & 6.9 & $\mathrm{~A} 0 \mathrm{Sr}$ & 2453074.840 & $1 \pm 51$ & \\
\hline 98340 & 55181 & 7.1 & B9 Si & 2453074.862 & $1033 \pm 65$ & ND \\
\hline \multirow[t]{2}{*}{99563} & 55890 & 8.5 & $\mathrm{~F} 0 \mathrm{Sr}$ & 2453012.747 & $-392 \pm 124$ & $\mathrm{CD}$ \\
\hline & & & & 2453015.725 & $-669 \pm 159$ & \\
\hline 105379 & 59167 & 8.0 & $\mathrm{~A} 0 \mathrm{SrCr}$ & 2453011.750 & $25 \pm 83$ & \\
\hline 105382 & 59173 & 4.5 & B6 He & 2453011.695 & $-1000 \pm 101$ & ND \\
\hline
\end{tabular}


O. Kochukhov and S. Bagnulo: Evolutionary state of magnetic chemically peculiar stars, Online Material p 3

Table 1. continued.

\begin{tabular}{|c|c|c|c|c|c|c|}
\hline HD & "HIP & $\bar{V}$ & Sp. type & JD & $\left\langle B_{z}\right\rangle(\mathrm{G})$ & Comment \\
\hline & & & & 2453015.746 & $-610 \pm 136$ & \\
\hline \multirow[t]{2}{*}{105770} & 59404 & 7.4 & B9 Si & 2453011.733 & $449 \pm 110$ & ND \\
\hline & & & & 2453120.645 & $262 \pm 71$ & \\
\hline 105999 & 59487 & 7.4 & $\mathrm{~F} 1 \mathrm{SrCr}$ & 2453011.770 & $-30 \pm 128$ & \\
\hline \multirow[t]{2}{*}{107696} & 60379 & 5.4 & $\mathrm{~B} 8 \mathrm{Cr}$ & 2452824.530 & $-46 \pm 108$ & \\
\hline & & & & 2453074.875 & $-75 \pm 142$ & \\
\hline 108945 & 61071 & 5.5 & A3 Sr & 2453015.835 & $65 \pm 116$ & \\
\hline 114365 & 64320 & 6.1 & $\mathrm{~A} 0 \mathrm{Si}$ & 2452824.543 & $8 \pm 100$ & \\
\hline 115226 & 64883 & 8.5 & A3 Sr & 2453086.799 & $677 \pm 57$ & ND \\
\hline 115440 & 65053 & 8.2 & B9 Si & 2453077.714 & $3217 \pm 61$ & ND \\
\hline 116890 & 65755 & 6.2 & B9 $\mathrm{Si}$ & 2452824.555 & $-292 \pm 77$ & ND \\
\hline \multirow[t]{2}{*}{117025} & 65783 & 6.1 & $\mathrm{~A} 2 \mathrm{SrCrEu}$ & 2452824.567 & $483 \pm 84$ & ND \\
\hline & & & & 2453120.664 & $463 \pm 68$ & \\
\hline \multirow[t]{2}{*}{118913} & 66888 & 7.7 & $\mathrm{~A} 0 \mathrm{SrCrEu}$ & 2452824.581 & $-345 \pm 88$ & ND \\
\hline & & & & 2453120.681 & $-555 \pm 38$ & \\
\hline 119308 & 66942 & 7.8 & $\mathrm{~A} 0 \mathrm{SrCrEu}$ & 2453120.704 & $-326 \pm 61$ & ND \\
\hline 122970 & 68790 & 8.3 & $\mathrm{~F} 0 \mathrm{SrCrEu}$ & 2453015.850 & $526 \pm 137$ & $\mathrm{CD}$ \\
\hline \multirow[t]{2}{*}{125630} & 70346 & 6.8 & $\mathrm{~A} 2 \mathrm{SiCrSr}$ & 2452824.607 & $660 \pm 67$ & ND \\
\hline & & & & 2453120.721 & $30 \pm 55$ & \\
\hline 127453 & 71314 & 7.4 & B8 Si & 2452824.621 & $-361 \pm 85$ & ND \\
\hline 127575 & 71359 & 7.7 & B9 $\mathrm{Si}$ & 2453079.888 & $911 \pm 64$ & ND \\
\hline 128775 & 71727 & 6.6 & B9 Si & 2453120.736 & $-278 \pm 52$ & ND \\
\hline 128974 & 71783 & 5.7 & $\mathrm{~A} 0 \mathrm{Si}$ & 2452824.644 & $-43 \pm 55$ & \\
\hline 129899 & 72670 & 6.4 & A0 Si & 2453120.795 & $495 \pm 42$ & ND \\
\hline \multirow[t]{2}{*}{130158} & 72323 & 5.6 & B9 Si & 2452824.676 & $2 \pm 53$ & \\
\hline & & & & 2453116.812 & $9 \pm 45$ & \\
\hline \multirow[t]{2}{*}{130557} & 72449 & 6.1 & B9 $\mathrm{SiCr}$ & 2452853.558 & $-10 \pm 70$ & \\
\hline & & & & 2453144.767 & $19 \pm 43$ & \\
\hline \multirow[t]{3}{*}{131120} & 72800 & 5.0 & B7 He-weak & 2452824.660 & $-152 \pm 114$ & \\
\hline & & & & 2453020.857 & $57 \pm 77$ & \\
\hline & & & & 2453030.864 & $152 \pm 125$ & \\
\hline 132322 & 73520 & 7.4 & $\mathrm{~A} 7 \mathrm{SrCrEu}$ & 2453111.811 & $340 \pm 40$ & ND \\
\hline \multirow[t]{2}{*}{133792} & 74181 & 6.3 & $\mathrm{~A} 0 \mathrm{SrCr}$ & 2452853.570 & $119 \pm 77$ & $\mathrm{CD}$ \\
\hline & & & & 2453120.812 & $124 \pm 40$ & \\
\hline 134305 & 74109 & 7.2 & A6 $\mathrm{SrCrEu}$ & 2453144.801 & $170 \pm 49$ & ND \\
\hline 136933 & 75439 & 5.4 & A0 Si & 2452823.720 & $23 \pm 89$ & \\
\hline 138758 & 76767 & 7.9 & B9 Si & 2453086.828 & $430 \pm 41$ & ND \\
\hline 138764 & 76243 & 5.2 & $\mathrm{~B} 6 \mathrm{Si}$ & 2452904.515 & $202 \pm 95$ & \\
\hline \multirow[t]{3}{*}{138769} & 76371 & 4.5 & B3 He & 2452904.503 & $91 \pm 95$ & \\
\hline & & & & 2452904.527 & $123 \pm 91$ & \\
\hline & & & & 2452908.522 & $-166 \pm 120$ & \\
\hline 145102 & 79235 & 6.6 & B9 Si & 2452763.815 & $40 \pm 79$ & \\
\hline \multirow[t]{2}{*}{147869} & 80351 & 5.8 & $\mathrm{~A} 1 \mathrm{Sr}$ & 2452763.827 & $53 \pm 74$ & \\
\hline & & & & 2453144.818 & $7 \pm 40$ & \\
\hline 148112 & 80463 & 4.6 & $\mathrm{~A} 0 \mathrm{CrEu}$ & 2452763.838 & $-59 \pm 64$ & \\
\hline 148898 & 80975 & 4.4 & $\mathrm{~A} 6 \mathrm{SrCrEu}$ & 2452763.849 & $241 \pm 84$ & \\
\hline \multirow[t]{2}{*}{149764} & 81477 & 6.9 & A0 Si & 2452763.874 & $-1169 \pm 86$ & ND \\
\hline & & & & 2453120.831 & $49 \pm 48$ & \\
\hline 149822 & 81337 & 6.4 & B9 SiCr & 2452763.861 & $-657 \pm 66$ & ND \\
\hline \multirow[t]{3}{*}{150549} & 82129 & 5.1 & A0 Si & 2452763.886 & $-167 \pm 63$ & \\
\hline & & & & 2453116.886 & $-52 \pm 60$ & \\
\hline & & & & 2453120.850 & $-49 \pm 34$ & \\
\hline \multirow[t]{2}{*}{151525} & 82216 & 5.2 & B9 $\mathrm{Eu} \mathrm{Cr}$ & 2452733.895 & $76 \pm 73$ & \\
\hline & & & & 2452763.897 & $237 \pm 75$ & $\mathrm{CD}$ \\
\hline 154708 & 84017 & 8.8 & $\mathrm{~A} 2 \mathrm{SrCrEu}$ & 2453120.876 & $6859 \pm 58$ & $\mathrm{CD}$ \\
\hline \multirow[t]{2}{*}{157751} & 85372 & 7.6 & B9 $\mathrm{SiCr}$ & 2452793.771 & $4070 \pm 65$ & ND \\
\hline & & & & 2453116.904 & $3982 \pm 48$ & \\
\hline 160468 & 86930 & 7.3 & $\mathrm{~F} 2 \mathrm{SrCr}$ & 2453116.862 & $-96 \pm 83$ & \\
\hline & & & & 2453134.819 & $-55 \pm 54$ & \\
\hline 161277 & 86983 & 7.1 & B9 $\mathrm{Si}$ & 2453134.840 & $94 \pm 44$ & \\
\hline
\end{tabular}


O. Kochukhov and S. Bagnulo: Evolutionary state of magnetic chemically peculiar stars, Online Material p 4

Table 1. continued.

\begin{tabular}{llrlrrr}
\hline \hline HD & HIP & $V$ & Sp. type & \multicolumn{1}{c}{ JD } & \multicolumn{1}{|c}{$\left\langle B_{z}\right\rangle(\mathrm{G})$} & Comment \\
\hline 166469 & 89178 & 6.5 & A0 SiCr Sr & 2452793.791 & $-42 \pm 49$ & \\
& & & & 2453136.772 & $-26 \pm 45$ & \\
168856 & 90030 & 7.0 & B9 Si & 2453144.840 & $-530 \pm 59$ & ND \\
171184 & 91001 & 8.0 & A0 Si & 2452880.529 & $250 \pm 52$ & ND \\
& & & & 2453144.868 & $-14 \pm 48$ & \\
171279 & 91031 & 7.3 & A0 SrCrEu & 2453144.893 & $-40 \pm 40$ & \\
172032 & 91414 & 7.7 & A9 SrCr & 2453151.605 & $-31 \pm 55$ & \\
172690 & 93481 & 7.5 & A0 Si SrCr & 2452793.814 & $-287 \pm 86$ & ND \\
& & & & 2453134.868 & $235 \pm 52$ & \\
175744 & 92934 & 6.6 & B9 Si & 2452880.555 & $104 \pm 76$ & \\
& & & & 2452901.519 & $162 \pm 91$ & \\
176196 & 93863 & 7.5 & B9 Eu Cr & 2452793.829 & $240 \pm 83$ & ND \\
& & & & 2453134.889 & $190 \pm 51$ & \\
183806 & 96178 & 5.6 & A0 CrEu Sr & 2452793.845 & $-23 \pm 64$ & ND \\
& & & & 2453120.924 & $148 \pm 37$ & \\
186117 & 97533 & 7.3 & A0 SrCrEu & 2453134.913 & $-19 \pm 49$ & \\
& & & & 2453140.829 & $27 \pm 46$ & \\
192674 & 100090 & 7.5 & B9 Cr Eu Sr & 2453137.861 & $7 \pm 44$ & \\
199180 & 103246 & 7.7 & A0 SiCr & 2452822.844 & $-228 \pm 85$ & \\
199728 & 103616 & 6.2 & B9 Si & 2452822.857 & $-245 \pm 73$ & ND \\
201018 & 104337 & 8.6 & A2 CrEu & 2453151.871 & $546 \pm 43$ & ND \\
202627 & 105140 & 4.7 & A1 Si & 2452793.874 & $-56 \pm 68$ & \\
206653 & 107525 & 7.2 & B9 Si & 2452793.894 & $32 \pm 68$ & \\
212385 & 110624 & 6.8 & A3 SrCrEu & 2452822.913 & $163 \pm 72$ & ND \\
& & & & 2453184.797 & $626 \pm 52$ & \\
221760 & 116389 & 4.7 & A2 SrCrEu & 2452793.915 & $-48 \pm 97$ & \\
& & & & 2453184.814 & $62 \pm 65$ & \\
\hline
\end{tabular}


O. Kochukhov and S. Bagnulo: Evolutionary state of magnetic chemically peculiar stars, Online Material p 5

Table 2. Fundamental parameters of magnetic CP stars. The columns give numbers in the HD and Hipparcos catalogues, distance determined from the Hipparcos parallax, absolute magnitude, $T_{\text {eff }}$, luminosity and mass in solar units, absolute and fractional stellar age. For the last two columns numbers in brackets give $1 \sigma$ ranges compatible with the errors of $T_{\text {eff }}$ and $L / L_{\odot}$.

\begin{tabular}{|c|c|c|c|c|c|c|c|c|}
\hline HD & HIP & $d(\mathrm{pc})$ & $\overline{M_{V}}$ & $\overline{\log T_{\text {eff }}(\mathrm{K})}$ & $\log L / L_{\odot}$ & $\bar{M} / M_{\odot}$ & $\log t(\mathrm{yr})$ & $\bar{\tau}$ \\
\hline 1048 & 1193 & $108 \pm 8$ & $1.05 \pm 0.17$ & $3.949 \pm 0.015$ & $1.50 \pm 0.07$ & $2.17 \pm 0.06$ & $8.72(8.63-8.79)$ & $0.61(0.49-0.71)$ \\
\hline 2453 & 2243 & $151 \pm 18$ & $0.88 \pm 0.27$ & $3.949 \pm 0.015$ & $1.57 \pm 0.11$ & $2.24 \pm 0.10$ & $8.72(8.65-8.78)$ & $0.68(0.54-0.78)$ \\
\hline 3980 & 3277 & $65 \pm 2$ & $1.62 \pm 0.09$ & $3.917 \pm 0.011$ & $1.24 \pm 0.04$ & $1.91 \pm 0.03$ & $8.83(8.75-8.89)$ & $0.53(0.45-0.61)$ \\
\hline 4778 & 3919 & $90 \pm 6$ & $1.23 \pm 0.15$ & $3.999 \pm 0.013$ & $1.51 \pm 0.07$ & $2.29 \pm 0.06$ & $8.26(7.53-8.47)$ & $0.24(0.03-0.40)$ \\
\hline 5737 & 4577 & $206 \pm 35$ & $-2.31 \pm 0.38$ & $4.121 \pm 0.013$ & $3.19 \pm 0.15$ & $5.01 \pm 0.21$ & $7.97(7.92-8.01)$ & $1.00(1.00-1.00)$ \\
\hline 8441 & 6560 & $203 \pm 33$ & $0.17 \pm 0.35$ & $3.956 \pm 0.014$ & $1.86 \pm 0.14$ & $2.57 \pm 0.18$ & $8.66(8.61-8.71)$ & $0.85(0.76-0.93)$ \\
\hline 9996 & 7651 & $139 \pm 16$ & $0.80 \pm 0.26$ & $4.012 \pm 0.013$ & $1.70 \pm 0.11$ & $2.50 \pm 0.10$ & $8.41(8.16-8.51)$ & $0.44(0.23-0.60)$ \\
\hline 10221 & 7965 & $136 \pm 11$ & $-0.21 \pm 0.18$ & $4.030 \pm 0.016$ & $2.15 \pm 0.08$ & $3.05 \pm 0.11$ & $8.42(8.37-8.46)$ & $0.79(0.69-0.86)$ \\
\hline 10783 & 8210 & $186 \pm 25$ & $0.08 \pm 0.30$ & $4.006 \pm 0.013$ & $1.98 \pm 0.12$ & $2.79 \pm 0.15$ & $8.51(8.47-8.55)$ & $0.76(0.65-0.85)$ \\
\hline 11187 & 8643 & $234 \pm 44$ & $0.14 \pm 0.41$ & $4.029 \pm 0.016$ & $2.00 \pm 0.17$ & $2.87 \pm 0.20$ & $8.43(8.33-8.49)$ & $0.68(0.48-0.82)$ \\
\hline 11503 & 8832 & $62 \pm 3$ & $0.60 \pm 0.12$ & $4.010 \pm 0.013$ & $1.78 \pm 0.05$ & $2.57 \pm 0.06$ & $8.47(8.38-8.54)$ & $0.55(0.44-0.65)$ \\
\hline 12288 & 9604 & $230 \pm 38$ & $0.59 \pm 0.36$ & $3.996 \pm 0.013$ & $1.75 \pm 0.15$ & $2.51 \pm 0.15$ & $8.54(8.42-8.60)$ & $0.61(0.42-0.75)$ \\
\hline 12447 & 9487 & $42 \pm 1$ & $1.03 \pm 0.10$ & $3.999 \pm 0.013$ & $1.58 \pm 0.05$ & $2.36 \pm 0.05$ & $8.41(8.21-8.53)$ & $0.37(0.23-0.49)$ \\
\hline 12767 & 9677 & $110 \pm 9$ & $-0.56 \pm 0.19$ & $4.111 \pm 0.013$ & $2.47 \pm 0.08$ & $3.75 \pm 0.13$ & $8.13(8.07-8.18)$ & $0.70(0.59-0.79)$ \\
\hline 14437 & 10951 & $197 \pm 36$ & $0.51 \pm 0.40$ & $4.034 \pm 0.016$ & $1.87 \pm 0.16$ & $2.72 \pm 0.17$ & 8.36 & $0.50(0.21-0.69)$ \\
\hline 15089 & 11569 & $43 \pm 1$ & $1.46 \pm 0.08$ & $3.925 \pm 0.010$ & $1.31 \pm 0.03$ & $1.97 \pm 0.03$ & $-8.86)$ & $0.56(0.48-0.62)$ \\
\hline 15144 & 11348 & $65 \pm 4$ & $1.91 \pm 0.14$ & $3.926 \pm 0.010$ & $1.13 \pm 0.06$ & $1.84 \pm 0.04$ & $8.63(8.30-8.77)$ & $0.30(0.13-0.42)$ \\
\hline 17775 & 13507 & $156 \pm 23$ & $1.92 \pm 0.33$ & $3.930 \pm 0.015$ & $1.13 \pm 0.13$ & $1.85 \pm 0.09$ & $-8.81)$ & $0.26(0.00-0.50)$ \\
\hline 18296 & 13775 & $118 \pm 12$ & $-0.44 \pm 0.23$ & $4.036 \pm 0.016$ & $2.25 \pm 0.10$ & $3.21 \pm 0.15$ & 8.38 & $0.74-0.90)$ \\
\hline 18610 & 13534 & $202 \pm 27$ & $1.55 \pm 0.30$ & $3.878 \pm 0.012$ & $1.27 \pm 0.12$ & $1.88 \pm 0.11$ & $-9.04)$ & $0.65-0.84)$ \\
\hline 19712 & 14376 & $166 \pm 25$ & $1.16 \pm 0.33$ & $4.056 \pm 0.015$ & $1.66 \pm 0.14$ & $2.61 \pm 0.11$ & $5-8.04)$ & $0.00(0.00-0.22)$ \\
\hline 19805 & 14980 & $168 \pm 24$ & $1.41 \pm 0.32$ & $3.973 \pm 0.014$ & $1.39 \pm 0.13$ & $2.13 \pm 0.10$ & $8.43(7.05-8.63)$ & $0.29(0.00-0.51)$ \\
\hline 19832 & 14893 & $113 \pm 11$ & $0.35 \pm 0.22$ & $4.095 \pm 0.014$ & $2.07 \pm 0.09$ & $3.17 \pm 0.12$ & $-8.16)$ & $0.29(0.06-0.49)$ \\
\hline 21699 & 16470 & $179 \pm 22$ & $-1.05 \pm 0.28$ & $4.159 \pm 0.012$ & $2.78 \pm 0.12$ & $4.48 \pm 0.22$ & 7.96 & $0.62-0.83)$ \\
\hline 22316 & 16974 & $170 \pm 20$ & $0.04 \pm 0.26$ & $4.073 \pm 0.015$ & $2.14 \pm 0.11$ & $3.16 \pm 0.13$ & $-8.32)$ & $0.58(0.39-0.71)$ \\
\hline 22374 & 16859 & $134 \pm 17$ & $0.82 \pm 0.28$ & $3.938 \pm 0.015$ & $1.58 \pm 0.11$ & $2.24 \pm 0.12$ & $8.76(8.71-8.81)$ & $0.74(0.62-0.83)$ \\
\hline 22470 & 16803 & $145 \pm 17$ & $-0.38 \pm 0.26$ & $4.115 \pm 0.013$ & $2.41 \pm 0.11$ & $3.67 \pm 0.15$ & $8.10(7.99-8.16)$ & $0.62(0.46-0.74)$ \\
\hline 22920 & 17167 & $226 \pm 38$ & $-1.32 \pm 0.37$ & $4.142 \pm 0.013$ & $2.85 \pm 0.15$ & $4.54 \pm 0.32$ & 8.01 & $.74-0.93)$ \\
\hline 23207 & 17345 & $177 \pm 31$ & $1.28 \pm 0.38$ & $3.896 \pm 0.011$ & $1.38 \pm 0.15$ & $2.00 \pm 0.14$ & $-8.97)$ & $0.64-0.85)$ \\
\hline 23408 & 17573 & $110 \pm 12$ & $-1.54 \pm 0.25$ & $4.079 \pm 0.014$ & $2.79 \pm 0.10$ & $4.22 \pm 0.18$ & $8.15(8.12-8.18)$ & $0.97(0.92-1.00)$ \\
\hline 24155 & 18033 & $135 \pm 16$ & $0.32 \pm 0.27$ & $4.132 \pm 0.013$ & $2.17 \pm 0.11$ & $3.45 \pm 0.14$ & $7.30(6.27-7.88)$ & $0.08(0.00-0.32)$ \\
\hline 24188 & 17543 & $142 \pm 10$ & $0.40 \pm 0.17$ & $4.101 \pm 0.014$ & $2.06 \pm 0.07$ & $3.19 \pm 0.10$ & $7.81(6.67-8.07)$ & $0.21(0.01-0.40)$ \\
\hline 24712 & 18339 & $48 \pm 2$ & $2.54 \pm 0.09$ & $3.857 \pm 0.012$ & $0.87 \pm 0.04$ & $1.55 \pm 0.03$ & $-9.17)$ & $.37-0.61)$ \\
\hline 25267 & 18673 & $101 \pm 7$ & $-0.30 \pm 0.15$ & $4.080 \pm 0.014$ & $2.30 \pm 0.07$ & $3.38 \pm 0.10$ & $-8.29)$ & $0.70(0.59-0.78)$ \\
\hline 25354 & 18912 & $144 \pm 20$ & $1.79 \pm 0.31$ & $3.993 \pm 0.013$ & $1.27 \pm 0.13$ & $2.12 \pm 0.08$ & $7.05(7.02-8.05)$ & $0.00(0.00-0.11)$ \\
\hline 25823 & 19171 & $151 \pm 19$ & $-0.63 \pm 0.28$ & $4.112 \pm 0.013$ & $2.50 \pm 0.12$ & $3.80 \pm 0.19$ & $8.13(8.07-8.17)$ & $0.72(0.59-0.82)$ \\
\hline 27309 & 20186 & $96 \pm 7$ & $0.40 \pm 0.16$ & $4.079 \pm 0.014$ & $2.01 \pm 0.07$ & $3.04 \pm 0.09$ & $3-8.23)$ & $0.34(0.15-0.51)$ \\
\hline 28843 & 21192 & $131 \pm 14$ & $0.06 \pm 0.24$ & $4.143 \pm 0.013$ & $2.30 \pm 0.10$ & $3.67 \pm 0.14$ & $-7.93)$ & $0.21(0.00-0.43)$ \\
\hline 30466 & 22402 & $163 \pm 25$ & $0.68 \pm 0.34$ & $4.044 \pm 0.016$ & $1.82 \pm 0.14$ & $2.71 \pm 0.14$ & $8.21(7.40-8.40)$ & $0.34(0.04-0.57)$ \\
\hline 32633 & 23733 & $156 \pm 22$ & $0.72 \pm 0.32$ & $4.108 \pm 0.014$ & $1.95 \pm 0.13$ & $3.10 \pm 0.13$ & $6.39(6.33-7.82)$ & $0.00(0.00-0.21)$ \\
\hline 34452 & 24799 & $137 \pm 13$ & $-0.33 \pm 0.21$ & $4.160 \pm 0.012$ & $2.49 \pm 0.09$ & $4.02 \pm 0.14$ & $7.82(7.51-7.94)$ & $0.40(0.19-0.55)$ \\
\hline 34797 & 24827 & $238 \pm 47$ & $-0.47 \pm 0.43$ & $4.102 \pm 0.014$ & $2.41 \pm 0.17$ & $3.62 \pm 0.26$ & $8.16(\varepsilon$ & $0.69(0.49-0.82)$ \\
\hline 38823 & 27423 & $113 \pm 12$ & $2.00 \pm 0.24$ & $3.839 \pm 0.013$ & $1.09 \pm 0.10$ & $1.70 \pm 0.08$ & $9.16(9.11-9.21)$ & $0.80(0.71-0.88)$ \\
\hline 39317 & 27743 & $157 \pm 22$ & $-0.50 \pm 0.31$ & $4.018 \pm 0.013$ & $2.24 \pm 0.13$ & $3.16 \pm 0.19$ & $8.42(8.37-8.47)$ & $0.88(0.80-0.93)$ \\
\hline 40312 & 28380 & $53 \pm 2$ & $-1.01 \pm 0.10$ & $4.007 \pm 0.013$ & $2.42 \pm 0.05$ & $3.41 \pm 0.07$ & $8.36(8.34-8.39)$ & $1.00(0.94-1.00)$ \\
\hline 42616 & 29565 & $173 \pm 28$ & $0.56 \pm 0.35$ & $3.989 \pm 0.013$ & $1.76 \pm 0.14$ & $2.50 \pm 0.15$ & $8.57(8.49-8.62)$ & $0.65(0.48-0.77)$ \\
\hline 42659 & 29365 & $135 \pm 14$ & $1.03 \pm 0.23$ & $3.900 \pm 0.011$ & $1.48 \pm 0.09$ & $2.10 \pm 0.10$ & $8.88(8.85-8.92)$ & $0.81(0.74-0.87)$ \\
\hline 49333 & 32504 & $204 \pm 30$ & $-0.51 \pm 0.32$ & $4.216 \pm 0.013$ & $2.69 \pm 0.13$ & $4.71 \pm 0.23$ & $7.27(6.07-7.67)$ & $0.16(0.00-0.43)$ \\
\hline 49976 & 32838 & $101 \pm 8$ & $1.20 \pm 0.18$ & $3.984 \pm 0.014$ & $1.49 \pm 0.07$ & $2.24 \pm 0.07$ & $8.45(8.14-8.59)$ & $0.35(0.16-0.51)$ \\
\hline 54118 & 34105 & $86 \pm 3$ & $0.38 \pm 0.09$ & $4.022 \pm 0.017$ & $1.89 \pm 0.05$ & $2.72 \pm 0.07$ & $8.45(8.34-8.52)$ & $0.61(0.49-0.70)$ \\
\hline 55522 & 34798 & $220 \pm 29$ & $-0.87 \pm 0.29$ & $4.211 \pm 0.013$ & $2.82 \pm 0.12$ & $4.88 \pm 0.22$ & $7.67(7.36-7.79)$ & $0.46(0.21-0.64)$ \\
\hline 55719 & 34802 & $133 \pm 8$ & $0.37 \pm 0.14$ & $3.960 \pm 0.014$ & $1.79 \pm 0.06$ & $2.49 \pm 0.07$ & $8.67(8.63-8.70)$ & $0.80(0.73-0.85)$ \\
\hline 56350 & 34929 & $161 \pm 12$ & $0.64 \pm 0.18$ & $4.022 \pm 0.017$ & $1.79 \pm 0.08$ & $2.61 \pm 0.09$ & $8.40(8.19-8.51)$ & $0.48(0.29-0.62)$ \\
\hline 60435 & 36537 & $233 \pm 45$ & $1.87 \pm 0.43$ & $3.910 \pm 0.011$ & $1.14 \pm 0.17$ & $1.82 \pm 0.12$ & $8.82(8.36-8.91)$ & $0.46(0.12-0.65)$ \\
\hline 62140 & 37934 & $81 \pm 4$ & $1.89 \pm 0.13$ & $3.884 \pm 0.011$ & $1.13 \pm 0.05$ & $1.77 \pm 0.04$ & $8.99(8.93-9.04)$ & $0.62(0.53-0.69)$ \\
\hline 63401 & 37982 & $210 \pm 24$ & $-0.40 \pm 0.26$ & $4.129 \pm 0.013$ & $2.45 \pm 0.11$ & $3.79 \pm 0.16$ & $8.03(7.90-8.11)$ & $0.58(0.41-0.71)$ \\
\hline 64486 & 39538 & $101 \pm 5$ & $0.32 \pm 0.12$ & $3.999 \pm 0.013$ & $1.87 \pm 0.05$ & $2.65 \pm 0.06$ & $8.54(8.49-8.59)$ & $0.70(0.62-0.77)$ \\
\hline
\end{tabular}


O. Kochukhov and S. Bagnulo: Evolutionary state of magnetic chemically peculiar stars, Online Material p 6

Table 2. continued.

\begin{tabular}{|c|c|c|c|c|c|c|c|c|}
\hline$\overline{\mathrm{HD}}$ & $\overline{\mathrm{HIP}}$ & $\overline{d(\mathrm{pc})}$ & $M_{V}$ & $\bar{l} \log T_{\text {eff }}(\mathrm{K})$ & $\log L / L_{\odot}$ & $M / M_{\odot}$ & $\log t(\mathrm{yr})$ & $\tau$ \\
\hline 64740 & 38500 & $220 \pm 25$ & $-2.15 \pm 0.25$ & $4.353 \pm 0.010$ & $3.63 \pm 0.10$ & $8.30 \pm 0.30$ & $7.10(6.79-7.24)$ & $0.40(0.18-0.57)$ \\
\hline 65339 & 39261 & $98 \pm 7$ & $1.12 \pm 0.17$ & $3.919 \pm 0.010$ & $1.45 \pm 0.07$ & $2.08 \pm 0.06$ & $8.84(8.81-8.87)$ & $0.72(0.64-0.78)$ \\
\hline 71866 & 41782 & $146 \pm 18$ & $0.83 \pm 0.28$ & $3.944 \pm 0.015$ & $1.58 \pm 0.11$ & $2.24 \pm 0.11$ & $8.74(8.68-8.79)$ & $0.71(0.58-0.81)$ \\
\hline 72968 & 42146 & $82 \pm 5$ & $1.11 \pm 0.15$ & $3.992 \pm 0.013$ & $1.54 \pm 0.07$ & $2.30 \pm 0.06$ & $8.43(8.18-8.55)$ & $0.36(0.19-0.50)$ \\
\hline 73340 & 42177 & $143 \pm 9$ & $-0.10 \pm 0.14$ & $4.145 \pm 0.012$ & $2.37 \pm 0.06$ & $3.77 \pm 0.11$ & $7.79(7.45-7.95)$ & $0.32(0.14-0.47)$ \\
\hline 74521 & 42917 & $125 \pm 13$ & $0.07 \pm 0.23$ & $4.033 \pm 0.016$ & $2.04 \pm 0.10$ & $2.92 \pm 0.12$ & $8.42(8.35-8.47)$ & $0.69(0.56-0.79)$ \\
\hline 75445 & 43257 & $113 \pm 8$ & $1.79 \pm 0.16$ & $3.885 \pm 0.011$ & $1.17 \pm 0.06$ & $1.80 \pm 0.05$ & $8.99(8.93-9.03)$ & $0.65(0.56-0.72)$ \\
\hline 79158 & 45290 & $175 \pm 25$ & $-0.94 \pm 0.31$ & $4.097 \pm 0.014$ & $2.59 \pm 0.13$ & $3.91 \pm 0.23$ & $8.16(8.12-8.20)$ & $0.84(0.74-0.92)$ \\
\hline 81009 & 45999 & $138 \pm 15$ & $1.17 \pm 0.24$ & $3.900 \pm 0.011$ & $1.42 \pm 0.10$ & $2.04 \pm 0.09$ & $8.90(8.86-8.94)$ & $0.78(0.69-0.84)$ \\
\hline 83368 & 47145 & $72 \pm 3$ & $1.91 \pm 0.12$ & $3.877 \pm 0.012$ & $1.12 \pm 0.05$ & $1.76 \pm 0.04$ & $9.02(8.96-9.07)$ & $0.65(0.56-0.72)$ \\
\hline 83625 & 47272 & $191 \pm 23$ & $0.35 \pm 0.26$ & $4.082 \pm 0.014$ & $2.04 \pm 0.11$ & $3.08 \pm 0.12$ & $8.07(7.63-8.24)$ & $0.36(0.12-0.56)$ \\
\hline 86199 & 48643 & $235 \pm 28$ & $-0.27 \pm 0.27$ & $4.112 \pm 0.013$ & $2.36 \pm 0.11$ & $3.58 \pm 0.15$ & $8.10(7.95-8.16)$ & $0.58(0.40-0.71)$ \\
\hline 88158 & 49642 & $250 \pm 32$ & $-0.72 \pm 0.28$ & $4.113 \pm 0.013$ & $2.54 \pm 0.12$ & $3.87 \pm 0.20$ & $8.12(8.08-8.16)$ & $0.75(0.63-0.84)$ \\
\hline 88385 & 49791 & $273 \pm 47$ & $0.66 \pm 0.38$ & $4.030 \pm 0.016$ & $1.80 \pm 0.16$ & $2.64 \pm 0.15$ & $8.34(7.88-8.47)$ & $0.43(0.13-0.64)$ \\
\hline 89103 & 50248 & $203 \pm 27$ & $1.19 \pm 0.29$ & $4.069 \pm 0.015$ & $1.67 \pm 0.12$ & $2.68 \pm 0.11$ & $6.68(6.59-7.64)$ & $0.00-0.08)$ \\
\hline 90044 & 50885 & $107 \pm 8$ & $0.74 \pm 0.17$ & $4.002 \pm 0.013$ & $1.71 \pm 0.07$ & $2.48 \pm 0.07$ & $8.49(8.36-8.56)$ & $0.52(0.38-0.63)$ \\
\hline 90569 & 51213 & $118 \pm 11$ & $0.64 \pm 0.21$ & $4.003 \pm 0.013$ & $1.75 \pm 0.09$ & $2.52 \pm 0.09$ & $8.50(8.39-8.57)$ & $0.56(0.42-0.68)$ \\
\hline 92385 & 52059 & $147 \pm 12$ & $0.81 \pm 0.18$ & $4.043 \pm 0.016$ & $1.77 \pm 0.08$ & $2.66 \pm 0.09$ & $8.12(7.28-8.35)$ & $0.26(0.03-0.46)$ \\
\hline 92499 & 52218 & $224 \pm 44$ & $2.10 \pm 0.44$ & $3.858 \pm 0.012$ & $1.05 \pm 0.17$ & $1.68 \pm 0.13$ & $9.10(9.02-9.14)$ & 0.68 \\
\hline 92664 & 52221 & $142 \pm 10$ & $-0.32 \pm 0.16$ & $4.155 \pm 0.012$ & $2.48 \pm 0.07$ & $3.97 \pm 0.12$ & $7.84(7.62-7.96)$ & $0.41(0.24-0.55)$ \\
\hline 94427 & 53290 & $110 \pm 11$ & $2.02 \pm 0.22$ & $3.861 \pm 0.012$ & $1.08 \pm 0.09$ & $1.71 \pm 0.07$ & 9.09 (9.04-9.13) & $0.69(0.59-0.78)$ \\
\hline 94660 & 53379 & $151 \pm 15$ & $0.20 \pm 0.22$ & $4.032 \pm 0.016$ & $1.98 \pm 0.09$ & $2.85 \pm 0.11$ & $8.42(8.33-8.48)$ & $0.64(0.50-0.75)$ \\
\hline 96707 & 54540 & $108 \pm 7$ & $0.87 \pm 0.14$ & $3.893 \pm 0.011$ & $1.54 \pm 0.06$ & $2.16 \pm 0.06$ & 8.87 & 0.80 \\
\hline 98088 & 55106 & $129 \pm 12$ & $0.79 \pm 0.21$ & $3.899 \pm$ & $1.57 \pm 0.09$ & $2.20 \pm 0.09$ & 8.85 & 91) \\
\hline 98340 & 55181 & $226 \pm 37$ & $0.86 \pm 0.35$ & $4.028 \pm 0.016$ & $1.71 \pm 0.15$ & $2.56 \pm 0.14$ & $8.24(6.82-8.45)$ & $0.32(0.00-0.56)$ \\
\hline 101065 & 56709 & $125 \pm 16$ & $2.48 \pm 0.29$ & $3.810 \pm 0.013$ & $0.91 \pm 0.12$ & $1.53 \pm 0.09$ & $9.32(9.25-9.40)$ & $0.86(0.76-0.94)$ \\
\hline 103192 & 57936 & $111 \pm 11$ & $-0.57 \pm 0.21$ & $4.044 \pm 0.016$ & $2.32 \pm 0.09$ & $3.33 \pm 0.14$ & 8.34( & $0.91)$ \\
\hline 105382 & 59173 & $115 \pm 9$ & $-0.93 \pm 0.18$ & $4.212 \pm$ & $2.85 \pm 0.08$ & 4.93 & 7.69( & 0.49 \\
\hline 105770 & 59404 & $194 \pm 23$ & $0.09 \pm 0.27$ & $4.115 \pm 0.013$ & $2.22 \pm 0.11$ & $3.43 \pm 0.14$ & $7.94(7.49-8.11)$ & $.12-0.55)$ \\
\hline 108662 & 60904 & $82 \pm 5$ & $0.57 \pm 0.15$ & $4.021 \pm 0.017$ & $1.81 \pm 0.07$ & $2.63 \pm 0.08$ & $8.42(8.26-8.52)$ & $0.52(0.35-0.65)$ \\
\hline 108945 & 61071 & $95 \pm 7$ & $0.54 \pm 0.17$ & $3.952 \pm 0.015$ & $1.71 \pm 0.07$ & $2.39 \pm 0.08$ & $8.70(8.66-8.74)$ & $0.78(0.69-0.84)$ \\
\hline 109026 & 61199 & $99 \pm 5$ & $-1.28 \pm 0.12$ & $4.173 \pm 0.012$ & $2.90 \pm 0.05$ & $4.79 \pm 0.12$ & $7.91(7.87-7.95)$ & $.71-0.83)$ \\
\hline 110066 & 61748 & $155 \pm 17$ & $0.41 \pm 0.25$ & $3.947 \pm$ & $1.75 \pm 0.10$ & $2.44 \pm 0.12$ & 8.71 & $0.82(0.73-0.89)$ \\
\hline 111133 & 62376 & $160 \pm 23$ & $0.20 \pm 0.33$ & $3.997 \pm 0.013$ & $1.92 \pm 0.13$ & $2.69 \pm 0.16$ & $8.55(8.5$ & $0.75(0.62-0.84)$ \\
\hline 112185 & 62956 & $24 \pm 0$ & $-0.21 \pm 0.04$ & $3.953 \pm 0.015$ & $2.01 \pm 0.02$ & $2.76 \pm 0.03$ & $8.61(8.59-8.63)$ & $0.94(0.90-0.97)$ \\
\hline 112381 & 63204 & $100 \pm 8$ & $1.53 \pm 0.19$ & $3.999 \pm 0.013$ & $1.39 \pm 0.08$ & $2.20 \pm 0.07$ & 7.02 & $0-0.22)$ \\
\hline 112413 & 63125 & $33 \pm 1$ & $0.26 \pm 0.08$ & $4.060 \pm 0$ & $2.03 \pm 0.05$ & $2.98 \pm 0.07$ & $8.27(8.14-8.36)$ & $0.52(0.39-0.63)$ \\
\hline 115226 & 64883 & $141 \pm 18$ & $2.57 \pm 0.29$ & $3.883 \pm 0.011$ & $0.86 \pm 0.12$ & $1.60 \pm 0.05$ & $8.60(7.37-9.16)$ & $0.18(0.00-0.62)$ \\
\hline 115440 & 65053 & $229 \pm 40$ & $0.81 \pm 0.39$ & $4.086 \pm 0.014$ & $1.86 \pm 0.16$ & $2.91 \pm 0.15$ & $6.51(6.45-8.04)$ & $0.00(0.00-0.30)$ \\
\hline 115708 & 64936 & $132 \pm 18$ & $2.19 \pm 0.31$ & $3.880 \pm 0.011$ & $1.01 \pm 0.12$ & $1.68 \pm 0.08$ & $8.97(8.76-9.04)$ & $0.50(0.28-0.65)$ \\
\hline 116114 & 65203 & $140 \pm 17$ & $1.41 \pm 0.28$ & $3.870 \pm 0.012$ & $1.32 \pm 0.11$ & $1.92 \pm 0.10$ & $9.01(8.96-9.05)$ & $0.74-0.89)$ \\
\hline 116458 & 65522 & $142 \pm 11$ & $-0.15 \pm 0.18$ & $4.012 \pm 0.013$ & $2.08 \pm 0.07$ & $2.93 \pm 0.10$ & $8.48(8.44-8.51)$ & $0.81(0.74-0.87)$ \\
\hline 116890 & 65755 & $214 \pm 26$ & $-0.94 \pm 0.27$ & $4.112 \pm 0.013$ & $2.63 \pm 0.11$ & $4.01 \pm 0.20$ & $8.12(8.08-8.15)$ & $0.81(0.72-0.89)$ \\
\hline 117025 & 65783 & $88 \pm 4$ & $1.25 \pm 0.13$ & $3.945 \pm 0.015$ & $1.42 \pm 0.05$ & $2.09 \pm 0.05$ & $8.72(8.61-8.80)$ & $0.55(0.43-0.65)$ \\
\hline 118022 & 66200 & $56 \pm 2$ & $1.17 \pm 0.10$ & $3.957 \pm 0.014$ & $1.46 \pm 0.04$ & $2.16 \pm 0.05$ & $.56-8.74)$ & $0.52(0.41-0.62)$ \\
\hline 118913 & 66888 & $206 \pm 31$ & $0.73 \pm 0.33$ & $3.981 \pm 0.014$ & $1.67 \pm 0.14$ & $2.40 \pm 0.14$ & $8.60(8.48-8.66)$ & $0.61(0.43-0.74)$ \\
\hline 119213 & 66700 & $88 \pm 4$ & $1.52 \pm 0.12$ & $3.941 \pm 0.015$ & $1.30 \pm 0.05$ & $1.99 \pm 0.05$ & $8.69(8.51-8.80)$ & $0.43(0.29-0.55)$ \\
\hline 119308 & 66942 & $182 \pm 31$ & $1.35 \pm 0.37$ & $4.009 \pm 0.013$ & $1.48 \pm 0.15$ & $2.30 \pm 0.11$ & $7.73(6.93-8.40)$ & $0.06(0.00-0.36)$ \\
\hline 119419 & 67036 & $112 \pm 9$ & $1.16 \pm 0.18$ & $4.048 \pm 0.016$ & $1.64 \pm 0.08$ & $2.55 \pm 0.09$ & $6.78(6.71-8.05)$ & $0.00(0.00-0.20)$ \\
\hline 120198 & 67231 & $86 \pm 4$ & $0.98 \pm 0.12$ & $4.023 \pm 0.016$ & $1.65 \pm 0.06$ & $2.49 \pm 0.07$ & $8.20(7.59-8.41)$ & $0.26(0.06-0.43)$ \\
\hline 122532 & 68673 & $169 \pm 21$ & $-0.15 \pm 0.28$ & $4.071 \pm 0.015$ & $2.22 \pm 0.12$ & $3.25 \pm 0.15$ & $8.27(8.18-8.33)$ & $0.67(0.51-0.78)$ \\
\hline 122970 & 68790 & $129 \pm 16$ & $2.74 \pm 0.28$ & $3.840 \pm 0.013$ & $0.79 \pm 0.11$ & $1.48 \pm 0.07$ & $9.20(8.99-9.29)$ & $0.57(0.33-0.71)$ \\
\hline 124224 & 69389 & $80 \pm 5$ & $0.46 \pm 0.16$ & $4.084 \pm 0.014$ & $2.00 \pm 0.07$ & $3.04 \pm 0.09$ & $7.97(7.45-8.18)$ & $0.27(0.07-0.45)$ \\
\hline 125248 & 69929 & $90 \pm 7$ & $1.20 \pm 0.18$ & $3.992 \pm 0.013$ & $1.50 \pm 0.08$ & $2.27 \pm 0.07$ & $8.37(7.94-8.53)$ & $0.30(0.10-0.47)$ \\
\hline 125630 & 70346 & $159 \pm 16$ & $0.55 \pm 0.23$ & $3.966 \pm 0.014$ & $1.72 \pm 0.10$ & $2.42 \pm 0.10$ & $8.66(8.61-8.70)$ & $0.73(0.62-0.81)$ \\
\hline 125823 & 70300 & $128 \pm 12$ & $-1.18 \pm 0.21$ & $4.248 \pm 0.012$ & $3.02 \pm 0.09$ & $5.57 \pm 0.20$ & $7.51(7.25-7.63)$ & $0.45(0.24-0.59)$ \\
\hline 126515 & 70553 & $141 \pm 21$ & $1.26 \pm 0.32$ & $4.007 \pm 0.013$ & $1.51 \pm 0.13$ & $2.32 \pm 0.11$ & $8.07(6.93-8.44)$ & $0.16(0.00-0.41)$ \\
\hline 127453 & 71314 & $257 \pm 49$ & $-0.17 \pm 0.42$ & $4.083 \pm 0.014$ & $2.25 \pm 0.17$ & $3.33 \pm 0.23$ & $8.22(8.07-8.28)$ & $0.64(0.41-0.79)$ \\
\hline 127575 & 71359 & $183 \pm 28$ & $0.91 \pm 0.34$ & $4.081 \pm 0.014$ & $1.81 \pm 0.14$ & $2.84 \pm 0.13$ & $6.55(6.49-7.98)$ & $0.00(0.00-0.24)$ \\
\hline 128775 & 71727 & $161 \pm 22$ & $0.48 \pm 0.30$ & $4.076 \pm 0.015$ & $1.97 \pm 0.12$ & $2.98 \pm 0.13$ & $8.04(7.12-8.25)$ & $0.30(0.03-0.53)$ \\
\hline
\end{tabular}


O. Kochukhov and S. Bagnulo: Evolutionary state of magnetic chemically peculiar stars, Online Material p 7

Table 2. continued.

\begin{tabular}{|c|c|c|c|c|c|c|c|c|}
\hline HD & HIP & $d(\mathrm{pc})$ & $M_{V}$ & $\bar{l} \log T_{\text {eff }}(\mathrm{K})$ & $\log L / L_{\odot}$ & $\bar{M} / M_{\odot}$ & $\log t(\mathrm{yr})$ & $\tau$ \\
\hline 128898 & 71908 & $16 \pm 0$ & $2.12 \pm 0.03$ & $3.885 \pm 0.011$ & $1.04 \pm 0.01$ & $1.71 \pm 0.02$ & $8.95(8.86-9.03)$ & $0.51(0.42-0.58)$ \\
\hline 129899 & 72670 & $275 \pm 43$ & $-0.94 \pm 0.34$ & $4.017 \pm 0.013$ & $2.41 \pm 0.14$ & $3.43 \pm 0.19$ & $8.36(8.31-8.41)$ & $0.95(0.89-1.00)$ \\
\hline 130559 & 72489 & $72 \pm 6$ & $1.32 \pm 0.20$ & $3.957 \pm 0.014$ & $1.40 \pm 0.08$ & $2.10 \pm 0.07$ & $8.62(8.42-8.72)$ & $0.44(0.26-0.58)$ \\
\hline 132322 & 73520 & $173 \pm 21$ & $0.93 \pm 0.28$ & $3.922 \pm 0.010$ & $1.52 \pm 0.11$ & $2.16 \pm 0.11$ & $8.82(8.78-8.86)$ & $0.76(0.67-0.83)$ \\
\hline 133029 & 73454 & $146 \pm 11$ & $0.48 \pm 0.18$ & $4.027 \pm 0.016$ & $1.86 \pm 0.08$ & $2.70 \pm 0.09$ & $8.41(8.26-8.49)$ & $0.54(0.37-0.67)$ \\
\hline 133652 & 73937 & $95 \pm 8$ & $0.81 \pm 0.20$ & $4.113 \pm 0.013$ & $1.93 \pm 0.09$ & $3.10 \pm 0.10$ & $6.39(6.34-7.21)$ & $0.00(0.00-0.04)$ \\
\hline 133792 & 74181 & $170 \pm 19$ & $-0.17 \pm 0.24$ & $3.974 \pm 0.014$ & $2.02 \pm 0.10$ & $2.80 \pm 0.14$ & $8.58(8.54-8.62)$ & $0.89(0.82-0.94)$ \\
\hline 133880 & 74066 & $126 \pm 13$ & $0.25 \pm 0.23$ & $4.079 \pm 0.014$ & $2.07 \pm 0.10$ & $3.10 \pm 0.12$ & $8.15(7.88-8.27)$ & $0.44(0.22-0.60)$ \\
\hline 134214 & 74145 & $91 \pm 7$ & $2.58 \pm 0.18$ & $3.858 \pm 0.012$ & $0.85 \pm 0.07$ & $1.55 \pm 0.04$ & $9.05(8.84-9.16)$ & $0.47(0.28-0.61)$ \\
\hline 134305 & 74109 & $177 \pm 27$ & $0.93 \pm 0.33$ & $3.908 \pm 0.011$ & $1.52 \pm 0.13$ & $2.15 \pm 0.14$ & $8.85(8.80-8.90)$ & $0.81(0.72-0.88)$ \\
\hline 137509 & 76011 & $249 \pm 37$ & $-0.28 \pm 0.33$ & $4.147 \pm 0.012$ & $2.44 \pm 0.14$ & $3.88 \pm 0.18$ & $7.89(7.51-8.02)$ & $0.43(0.17-0.62)$ \\
\hline 137909 & 75695 & $34 \pm 0$ & $1.12 \pm 0.06$ & $3.871 \pm 0.012$ & $1.44 \pm 0.02$ & $2.04 \pm 0.02$ & 8.95 & $0.88(0.84-0.91)$ \\
\hline 137949 & 75848 & $89 \pm 6$ & $1.80 \pm 0.17$ & $3.861 \pm 0.012$ & $1.16 \pm 0.07$ & $1.78 \pm 0.06$ & $9.07(9.03-9.11)$ & $0.75(0.68-0.82)$ \\
\hline 138758 & 76767 & $238 \pm 44$ & $0.92 \pm 0.41$ & $4.023 \pm 0.016$ & $1.68 \pm 0.17$ & $2.51 \pm 0.15$ & $8.26(6.86-8.47)$ & $0.31(0.00-0.57)$ \\
\hline 140160 & 76866 & $70 \pm 4$ & $1.04 \pm 0.13$ & $3.968 \pm 0.014$ & $1.53 \pm 0.06$ & $2.24 \pm 0.06$ & $8.62(8.52-8.70)$ & $0.53(0.41-0.63)$ \\
\hline 140728 & 76957 & $97 \pm 4$ & $0.51 \pm 0.11$ & $4.021 \pm 0.012$ & $1.84 \pm 0.05$ & $2.66 \pm 0.06$ & $8.43(\varepsilon$ & 0.55 \\
\hline 142301 & 77909 & $139 \pm 23$ & $-0.24 \pm 0.37$ & $4.193 \pm 0.011$ & $2.53 \pm 0.15$ & $4.30 \pm 0.21$ & 7.216 & 0.11 \\
\hline 142990 & 78246 & $149 \pm 18$ & $-0.74 \pm 0.27$ & $4.217 \pm 0.013$ & $2.78 \pm 0.11$ & $4.87 \pm 0.20$ & $7.54(6.98-7.73)$ & $0.34(0.08-0.54)$ \\
\hline 143473 & 78533 & $123 \pm 15$ & $1.06 \pm 0.27$ & $4.109 \pm 0.014$ & $1.82 \pm 0.11$ & $3.00 \pm 0.12$ & $6.44(6.38-6.52)$ & $0.00(0.00-0.00)$ \\
\hline 144334 & 78877 & $149 \pm 19$ & $-0.29 \pm 0.28$ & $4.168 \pm 0.012$ & $2.50 \pm 0.12$ & $4.08 \pm 0.17$ & $7.72(7.1$ & $0.33(0.07-0.52)$ \\
\hline 145501 & 79374 & $133 \pm 19$ & $0.03 \pm 0.33$ & $4.141 \pm 0$ & $2.31 \pm 0.13$ & $3.67 \pm 0.17$ & $7.72(6$. & 0.25 \\
\hline 147010 & 80024 & $143 \pm 19$ & $0.54 \pm 0.30$ & $4.117 \pm 0.013$ & $2.04 \pm 0.12$ & $3.23 \pm 0.14$ & $6.47(6$. & 0.00 \\
\hline 148112 & 80463 & $72 \pm 4$ & $0.20 \pm 0.15$ & $3.970 \pm 0.014$ & $1.87 \pm 0.06$ & $2.60 \pm 0.08$ & $8.63(8.59-8.66)$ & $0.81(0.75-0.87)$ \\
\hline 148199 & 80607 & $150 \pm 21$ & $0.49 \pm 0.31$ & $4.046 \pm 0.016$ & $1.90 \pm 0.13$ & $2.79 \pm 0.13$ & $8.29(7.95-8.40)$ & $0.45(0.20-0.64)$ \\
\hline 148330 & 80375 & $112 \pm 6$ & $0.49 \pm 0.12$ & $3.947 \pm 0$ & $1.72 \pm 0.05$ & $2.40 \pm 0.06$ & $8.71(8$ & 0.8 \\
\hline 149764 & 81477 & $126 \pm 15$ & $0.99 \pm 0.28$ & $4.128 \pm 0$ & $1.89 \pm 0.12$ & $3.19 \pm 0.12$ & $6.35(6$ & $.00)$ \\
\hline 149822 & 81337 & $133 \pm 14$ & $0.70 \pm 0.23$ & $4.010 \pm 0.013$ & $1.74 \pm 0.10$ & $2.53 \pm 0.09$ & $8.46(8$ & $0.33-0.64)$ \\
\hline 149911 & 81440 & $126 \pm 16$ & $0.06 \pm 0.29$ & $3.970 \pm 0.014$ & $1.93 \pm 0.12$ & $2.67 \pm 0.15$ & $8.61(8.57-8.65)$ & $0.85(0.76-0.92)$ \\
\hline 151525 & 82216 & $141 \pm 17$ & $-0.64 \pm 0.27$ & $3.971 \pm 0.014$ & $2.21 \pm 0.11$ & $3.04 \pm 0.13$ & $8.51(8.4$ & $0.92-1.00)$ \\
\hline 151965 & 82554 & $180 \pm 28$ & $-0.09 \pm 0.35$ & $4.154 \pm 0$ & $2.38 \pm 0.14$ & $3.85 \pm 0.19$ & $7.68(6$. & $0.00-0.50)$ \\
\hline 152107 & 82321 & $53 \pm 1$ & $1.21 \pm 0.07$ & $3.941 \pm 0$ & $1.43 \pm 0.03$ & $2.10 \pm 0.04$ & 8.74 & $0.48-0.67)$ \\
\hline 153882 & 83308 & $168 \pm 20$ & $-0.07 \pm 0.27$ & $3.988 \pm 0.013$ & $2.00 \pm 0.11$ & $2.79 \pm 0.14$ & 8.55 & $0.84(0.76-0.91)$ \\
\hline 154708 & 84017 & $140 \pm 22$ & $2.90 \pm 0.35$ & $3.829 \pm 0.013$ & $0.73 \pm 0.14$ & $1.43 \pm 0.08$ & $9.29(9.06-9.38)$ & $0.61(0.33-0.76)$ \\
\hline 157751 & 85372 & $164 \pm 28$ & $1.52 \pm 0.38$ & $3.993 \pm 0$ & $1.38 \pm 0.15$ & $2.18 \pm 0.10$ & 7.56 & 0.0 \\
\hline 164258 & 88148 & $121 \pm 13$ & $0.63 \pm 0.24$ & $3.952 \pm 0$ & $1.67 \pm 0.10$ & $2.35 \pm 0.11$ & $8.71(\varepsilon$ & 0.75 \\
\hline 165474 & 88627 & $130 \pm 20$ & $1.75 \pm 0.35$ & $3.885 \pm 0.011$ & $1.19 \pm 0.14$ & $1.82 \pm 0.11$ & 8.99 & $0.66(0.51-0.77)$ \\
\hline 168733 & 90074 & $189 \pm 27$ & $-1.14 \pm 0.31$ & $4.108 \pm 0.014$ & $2.70 \pm 0.13$ & $4.13 \pm 0.25$ & $8.12(8.08-8.16)$ & $0.87(0.78-0.94)$ \\
\hline 168856 & 90030 & $179 \pm 29$ & $0.28 \pm 0.37$ & $4.106 \pm 0.014$ & $2.13 \pm 0.15$ & $3.28 \pm 0.17$ & $7.89(6.34-8.14)$ & $0.28(0.00-0.54)$ \\
\hline 170000 & 89908 & $88 \pm 3$ & $-0.39 \pm 0.10$ & $4.058 \pm 0$ & $2.28 \pm 0.05$ & $3.30 \pm 0.08$ & $-8.36)$ & 0.7 \\
\hline 170397 & 90651 & $87 \pm 6$ & $1.14 \pm 0.16$ & $4.033 \pm 0.016$ & $1.61 \pm 0.07$ & $2.48 \pm 0.08$ & $7.70(6.7$ & $0.07(0.00-0.29)$ \\
\hline 171184 & 91001 & $186 \pm 35$ & $0.44 \pm 0.41$ & $4.081 \pm 0.014$ & $2.00 \pm 0.17$ & $3.03 \pm 0.18$ & $8.02(6.48-8.25)$ & $0.30(0.00-0.56)$ \\
\hline 171586 & 91142 & $102 \pm 9$ & $1.15 \pm 0.21$ & $3.967 \pm 0.014$ & $1.48 \pm 0.09$ & $2.19 \pm 0.08$ & $8.60(8.43-8.69)$ & $0.48(0.30-0.61)$ \\
\hline 172690 & 93481 & $249 \pm 42$ & $0.19 \pm 0.37$ & $4.058 \pm 0$ & $2.05 \pm 0.15$ & $3.00 \pm 0.18$ & $8.30(8$. & $0.32-0.72)$ \\
\hline 173650 & 92036 & $214 \pm 34$ & $-0.42 \pm 0.35$ & $4.015 \pm 0.013$ & $2.20 \pm 0.14$ & $3.10 \pm 0.20$ & $8.44(8.39-8.49)$ & $0.86(0.78-0.93)$ \\
\hline 175132 & 92599 & $374 \pm 72$ & $-1.72 \pm 0.43$ & $4.022 \pm 0.017$ & $2.73 \pm 0.17$ & $3.73 \pm 0.18$ & $8.27(8.23-8.32)$ & $1.00-1.00)$ \\
\hline 175362 & 92989 & $130 \pm 15$ & $-0.40 \pm 0.27$ & $4.217 \pm 0.013$ & $2.64 \pm 0.11$ & $4.64 \pm 0.20$ & $6.92(6.04-7.56)$ & $0.06(0.00-0.32)$ \\
\hline 176196 & 93863 & $255 \pm 44$ & $0.47 \pm 0.38$ & $4.000 \pm 0.013$ & $1.81 \pm 0.15$ & $2.58 \pm 0.17$ & $8.53(8.43-8.59)$ & $0.64(0.46-0.77)$ \\
\hline 176232 & 93179 & $74 \pm 3$ & $1.42 \pm 0.11$ & $3.899 \pm 0.011$ & $1.32 \pm 0.04$ & $1.95 \pm 0.04$ & $8.93(8.88-8.96)$ & $0.71(0.64-0.77)$ \\
\hline 179527 & 94311 & $300 \pm 49$ & $-1.64 \pm 0.36$ & $4.046 \pm 0.016$ & $2.75 \pm 0.15$ & $3.94 \pm 0.18$ & $8.22(8.18-8.26)$ & $1.00(0.96-1.00)$ \\
\hline 183056 & 95556 & $200 \pm 20$ & $-1.28 \pm 0.22$ & $4.086 \pm 0.014$ & $2.70 \pm 0.10$ & $4.08 \pm 0.18$ & $8.16(8.12-8.19)$ & $0.93(0.87-0.97)$ \\
\hline 183339 & 95520 & $384 \pm 76$ & $-1.35 \pm 0.43$ & $4.126 \pm 0.013$ & $2.82 \pm 0.18$ & $4.44 \pm 0.37$ & $8.05(7.99-8.10)$ & $0.88(0.78-0.96)$ \\
\hline 183806 & 96178 & $133 \pm 15$ & $-0.09 \pm 0.25$ & $3.983 \pm 0.014$ & $2.00 \pm 0.10$ & $2.79 \pm 0.14$ & $8.56(8.52-8.60)$ & $0.85(0.78-0.92)$ \\
\hline 184905 & 96292 & $165 \pm 13$ & $0.34 \pm 0.18$ & $4.035 \pm 0.016$ & $1.93 \pm 0.08$ & $2.80 \pm 0.09$ & $8.39(8.26-8.47)$ & $0.57(0.41-0.69)$ \\
\hline 187474 & 97749 & $103 \pm 9$ & $0.32 \pm 0.20$ & $4.004 \pm 0.013$ & $1.88 \pm 0.08$ & $2.67 \pm 0.09$ & $8.52(8.47-8.57)$ & $0.69(0.58-0.77)$ \\
\hline 188041 & 97871 & $84 \pm 6$ & $0.86 \pm 0.17$ & $3.926 \pm 0.010$ & $1.55 \pm 0.07$ & $2.20 \pm 0.07$ & $8.80(8.77-8.83)$ & $0.77(0.70-0.82)$ \\
\hline 192678 & 99672 & $230 \pm 27$ & $0.41 \pm 0.26$ & $3.987 \pm 0.013$ & $1.81 \pm 0.10$ & $2.56 \pm 0.12$ & $8.59(8.54-8.63)$ & $0.71(0.59-0.80)$ \\
\hline 196178 & 101475 & $147 \pm 14$ & $-0.16 \pm 0.22$ & $4.126 \pm 0.013$ & $2.35 \pm 0.09$ & $3.64 \pm 0.13$ & $7.99(7.76-8.09)$ & $0.47(0.27-0.61)$ \\
\hline 196502 & 101260 & $127 \pm 8$ & $-0.36 \pm 0.15$ & $3.964 \pm 0.014$ & $2.08 \pm 0.07$ & $2.87 \pm 0.09$ & $8.57(8.54-8.60)$ & $0.94(0.90-1.00)$ \\
\hline 199728 & 103616 & $131 \pm 17$ & $0.60 \pm 0.30$ & $4.078 \pm 0.015$ & $1.93 \pm 0.12$ & $2.95 \pm 0.13$ & $7.89(6.51-8.21)$ & $0.21(0.00-0.45)$ \\
\hline 200177 & 103658 & $139 \pm 11$ & $1.47 \pm 0.18$ & $3.997 \pm 0.013$ & $1.41 \pm 0.08$ & $2.21 \pm 0.07$ & $7.70(6.99-8.34)$ & $0.05(0.00-0.27)$ \\
\hline
\end{tabular}


O. Kochukhov and S. Bagnulo: Evolutionary state of magnetic chemically peculiar stars, Online Material $p 8$

Table 2. continued.

\begin{tabular}{ccccccccc}
\hline \hline HD & HIP & \multicolumn{1}{c}{$d(\mathrm{pc})$} & \multicolumn{1}{c}{$M_{V}$} & $\log T_{\text {eff }}(\mathrm{K})$ & $\log L / L_{\odot}$ & $M / M_{\odot}$ & $\log t(\mathrm{yr})$ & $\tau$ \\
\hline 201018 & 104337 & $174 \pm 32$ & $2.32 \pm 0.40$ & $3.941 \pm 0.015$ & $0.98 \pm 0.16$ & $1.82 \pm 0.07$ & $7.05(7.05-8.41)$ & $0.00(0.00-0.17)$ \\
201601 & 104521 & $35 \pm 1$ & $1.98 \pm 0.07$ & $3.882 \pm 0.011$ & $1.10 \pm 0.03$ & $1.74 \pm 0.03$ & $8.99(8.93-9.05)$ & $0.59(0.52-0.66)$ \\
203006 & 105382 & $57 \pm 2$ & $1.13 \pm 0.11$ & $3.989 \pm 0.013$ & $1.52 \pm 0.05$ & $2.28 \pm 0.05$ & $8.44(8.23-8.56)$ & $0.37(0.22-0.49)$ \\
204411 & 105898 & $119 \pm 7$ & $-0.10 \pm 0.14$ & $3.942 \pm 0.015$ & $1.95 \pm 0.06$ & $2.68 \pm 0.07$ & $8.65(8.62-8.68)$ & $0.94(0.89-0.98)$ \\
205087 & 106355 & $186 \pm 23$ & $0.20 \pm 0.28$ & $4.038 \pm 0.016$ & $2.00 \pm 0.12$ & $2.88 \pm 0.14$ & $8.39(8.28-8.46)$ & $0.63(0.45-0.75)$ \\
208217 & 108340 & $146 \pm 19$ & $1.50 \pm 0.29$ & $3.904 \pm 0.011$ & $1.29 \pm 0.12$ & $1.93 \pm 0.10$ & $8.91(8.85-8.95)$ & $0.66(0.52-0.76)$ \\
212385 & 110624 & $112 \pm 11$ & $1.58 \pm 0.23$ & $3.923 \pm 0.010$ & $1.26 \pm 0.09$ & $1.93 \pm 0.07$ & $8.80(8.69-8.86)$ & $0.51(0.37-0.63)$ \\
215038 & 111849 & $265 \pm 42$ & $0.57 \pm 0.35$ & $4.136 \pm 0.013$ & $2.08 \pm 0.14$ & $3.39 \pm 0.14$ & $6.29(6.26-7.60)$ & $0.00(0.00-0.16)$ \\
216018 & 112705 & $109 \pm 11$ & $2.51 \pm 0.24$ & $3.889 \pm 0.011$ & $0.88 \pm 0.10$ & $1.63 \pm 0.04$ & $8.54(7.29-8.90)$ & $0.16(0.00-0.40)$ \\
217522 & 113711 & $95 \pm 8$ & $2.62 \pm 0.20$ & $3.816 \pm 0.013$ & $0.85 \pm 0.08$ & $1.49 \pm 0.06$ & $9.33(9.27-9.40)$ & $0.80(0.69-0.87)$ \\
217833 & 113797 & $221 \pm 40$ & $-0.52 \pm 0.40$ & $4.171 \pm 0.012$ & $2.59 \pm 0.16$ & $4.23 \pm 0.24$ & $7.82(7.41-7.94)$ & $0.45(0.16-0.66)$ \\
220825 & 115738 & $49 \pm 1$ & $1.45 \pm 0.09$ & $3.958 \pm 0.014$ & $1.35 \pm 0.04$ & $2.07 \pm 0.04$ & $8.57(8.36-8.70)$ & $0.37(0.23-0.48)$ \\
221006 & 115908 & $116 \pm 7$ & $0.29 \pm 0.14$ & $4.135 \pm 0.013$ & $2.19 \pm 0.06$ & $3.49 \pm 0.10$ & $7.32(6.26-7.80)$ & $0.08(0.00-0.27)$ \\
221394 & 116119 & $147 \pm 14$ & $0.45 \pm 0.22$ & $3.979 \pm 0.014$ & $1.78 \pm 0.09$ & $2.51 \pm 0.10$ & $8.62(8.56-8.66)$ & $0.72(0.61-0.80)$ \\
221568 & 116210 & $243 \pm 39$ & $0.24 \pm 0.36$ & $3.962 \pm 0.014$ & $1.84 \pm 0.14$ & $2.56 \pm 0.17$ & $8.65(8.60-8.70)$ & $0.82(0.72-0.91)$ \\
223640 & 117629 & $98 \pm 10$ & $0.17 \pm 0.22$ & $4.089 \pm 0.014$ & $2.13 \pm 0.10$ & $3.20 \pm 0.12$ & $8.11(7.84-8.23)$ & $0.44(0.23-0.60)$ \\
224801 & 63 & $207 \pm 30$ & $-0.43 \pm 0.32$ & $4.073 \pm 0.015$ & $2.33 \pm 0.13$ & $3.41 \pm 0.20$ & $8.27(8.21-8.31)$ & $0.76(0.62-0.85)$ \\
\hline
\end{tabular}

